\title{
Path integrals for potential scattering
}

\author{
R. Rosenfelder* \\ Particle Theory Group, Paul Scherrer Institute, CH-5232 Villigen PSI, Switzerland
}

(Received 11 July 2008; published 6 January 2009)

\begin{abstract}
Two path integral representations for the $T$ matrix in nonrelativistic potential scattering are derived and proved to produce the complete Born series when expanded to all orders. They are obtained with the help of "phantom" degrees of freedom which take away explicit phases that diverge for asymptotic times. In addition, energy conservation is enforced by imposing a Faddeev-Popov-like constraint in the velocity path integral. These expressions may be useful for attempts to evaluate the path integral in real time and for alternative multiple scattering expansions. Standard eikonal-type high-energy approximations and systematic expansions immediately follow.
\end{abstract}

DOI: 10.1103/PhysRevA.79.012701

PACS number(s): 03.65.Nk, 03.65.Db

\section{INTRODUCTION}

Nonrelativistic quantum mechanical scattering in a local potential is usually described in the framework of timedependent or time-independent solutions of the Schrödinger equation (see, for example, Ref. [1]). Path-integral methods in quantum mechanics, on the other hand, are mostly applied to the discrete spectrum, e.g., for harmonic [2] or anharmonic oscillators, in particular for evaluating the energy splitting in the double-well potential [3]. In contrast, the transition matrix for the continuous spectrum is rarely represented as path integral. Even if available, many representations turn out to be rather formal, e.g., requiring infinitely many differentiations $[4,5]$ or infinite time limits to be performed $[6,7]$. This is not only impractible but also unfortunate since a convenient path integral representation may lead to new approximations and may be extended readily to the many-body problem or quantum field theory.

Also the long-standing problem how to evaluate real-time path integrals by stochastic methods needs a suitable path integral representation as starting method. There has been significant progress in dealing with real-time path integrals for dissipative systems [8] and with coherent-state path integrals for autocorrelation functions [9] but in closed systems and infinite scattering times only zero-energy scattering seems to be tractable by Euclidean Monte Carlo methods [10] at present (for other attempts, see Refs. [11,12]). Medium- and high-energy many-body scattering has to rely on multiple scattering expansions apart from the very restricted few-body cases where exact quantum mechanical calculations are possible [13].

One of the most simple and versatile multiple scattering versions-Glauber's approach-is based on the timehonored eikonal approximation where the particle is assumed to travel along a straight-line trajectory. This restricts the application usually to high-energy, forward scattering. For potential scattering systematic improvements to this approximation have been worked out long ago $[14,15]$ but even at high energies the convergence of these expansions is unsatisfactory for some classes of potentials. There are numerous

\footnotetext{
*roland.rosenfelder@psi.ch
}

other studies which try to extend the range of validity of the eikonal approximation (see, e.g., Ref. [16]). Clearly a path integral representation for the $T$ matrix which naturally gives rise to these high-energy approximations would be useful both for the analytical and (perhaps) for the numerical problems mentioned above.

As one of the merits of a path integral approach is its direct extension to field theory one may also expect applications in the relativistic domain where the usual procedure for an eikonal approximation consists of simplifying individual diagrams and resumming them [17].

In the present work we will derive a path-integral representation of the $T$ matrix in potential scattering which is similar to the one given by Campbell et al. [18] many years ago (see also Refs. [19,20]). However, ours is not a phasespace path integral as developed there but a particular path integral over velocities which is a significant reduction in complexity. The most obvious application is at high energy where a new sequence of high-energy approximations immediately follows. However, the main aim of the present work is not to give another high-energy approximation but to demonstrate that path integral methods lead to new, conceptually (albeit not technically) simple results which may be extended to the many-body case.

Preliminary results have already been presented elsewhere [21] and a (slightly different) account is included in a textbook on path integrals [22]. These previous attempts suffered from ambiguities in the limit of large scattering times where energy conservation and the elimination of "dangerous" phases from the $S$ matrix have to be achieved. In particular, the order in which these procedures were taken seemed to give many, at first sight equivalent formulations which however did not stand up to further scrutinity. In the present, detailed account we first eliminate these phases by introducing "phantom" degrees of freedom (dynamical variables with the wrong-sign kinetic term) and then isolate the variables whose large-time behavior gives rise to energy conservation by a suitable insertion of unity into the path integral. This is the classical Faddeev-Popov trick which first was utilized by Campbell et al. for path-integral descriptions of potential scattering. The resulting path-integral representation of the $T$ matrix is shown to be valid by explicitly working out the Born series to arbitrary order. We give two versions of this path-integral representation corresponding to different refer- 
ence paths (straight-line or eikonal and "ray") about which the quantum fluctuations have to evaluated.

This paper is organized as follows. In Sec. II we introduce velocity path integrals which are particularly suited for our purposes. Sections III and IV describe how "phantom" degrees of freedom naturally arise and the implementation of our particular Faddeev-Popov constraint. The "ray" representation is developed in Sec. V and systematic high-energy expansions are worked out in Sec. VI. These are tested numerically for scattering from a Gaussian potential in Sec. VII followed by our conclusions and outlook. More technical details can be found in three appendixes.

\section{VELOCITY PATH INTEGRALS FOR THE $S$ MATRIX}

Consider nonrelativistic scattering in a local potential $V(\mathbf{r})$ which vanishes asymptotically so that the corresponding Hamiltonian (also) has a continuous spectrum. The initial momentum of the particle with mass $m$ is $\mathbf{k}_{i}(\hbar=1)$ and the final momentum $\mathbf{k}_{f}$. Our scattering states are normalized according to $\left\langle\phi_{f} \mid \phi_{i}\right\rangle=(2 \pi)^{3} \delta^{(3)}\left(\mathbf{k}_{f}-\mathbf{k}_{i}\right)$. Time-dependent scattering is formulated in the interaction picture [1] in which the free propagation has been removed. The $S$ matrix is then just the matrix element of the time-evolution operator in the interaction picture

$$
\hat{U}_{I}\left(t_{b}, t_{a}\right)=e^{i \hat{H}_{0} t_{b}} \hat{U}\left(t_{b}, t_{a}\right) e^{-i \hat{H}_{0} t_{a}}, \quad \hat{U}\left(t_{b}, t_{a}\right)=\exp \left[-i \hat{H}\left(t_{b}-t_{a}\right)\right]
$$

taken between scattering states and evaluated at asymptotic times

$$
\begin{aligned}
S_{i \rightarrow f}= & \lim _{T \rightarrow \infty}\left\langle\phi_{f}\left|\hat{U}_{I}(T,-T)\right| \phi_{i}\right\rangle \\
= & \lim _{T \rightarrow \infty} e^{i\left(E_{i}+E_{f}\right) T}\left\langle\phi_{f}|\hat{U}(T,-T)| \phi_{i}\right\rangle \\
& =:(2 \pi)^{3} \delta^{(3)}\left(\mathbf{k}_{i}-\mathbf{k}_{f}\right)-2 \pi i \delta\left(E_{i}-E_{f}\right) T_{i \rightarrow f} .
\end{aligned}
$$

The second line defines the $T$ matrix after the energy conserving $\delta$ function has been factored out. Then $E_{i}=\mathbf{k}_{i}^{2} /(2 m)$ $=\mathbf{k}_{f}^{2} /(2 m)=E_{f}=k^{2} /(2 m) \equiv E$ is the common scattering energy.

To find a path integral representation of the $T$ matrix we start from the standard path integral expression for the matrix element of the time-evolution operator $U\left(\mathbf{x}_{b}, t_{b} ; \mathbf{x}_{a}, t_{a}\right)$ $\equiv\left\langle\mathbf{x}_{b}\left|\exp \left[-i \hat{H}\left(t_{b}-t_{a}\right)\right]\right| \mathbf{x}_{a}\right\rangle[2]$ in which one integrates functionally over all paths starting at $\mathbf{x}_{a}$ at time $t_{a}$ and ending at $\mathbf{x}_{b}$ at time $t_{b}$. As usual this is realized by dividing the time difference into $N$ intervals $\epsilon=\left(t_{b}-t_{a}\right) / N$ and integrating over all intermediate points $\mathbf{x}_{k}, k=1, \ldots N-1$ with the exponential of $i$ times the classical action as weight.

For our purposes it is, however, more convenient to integrate functionally over velocities $[20,23]$ which is achieved by multiplying the time sliced path integral for $U$ with the following factor:

$$
1=\prod_{k=1}^{N} \int d^{3} v_{k} \delta\left(\frac{\mathbf{x}_{k}-\mathbf{x}_{k-1}}{\epsilon}-\mathbf{v}_{k}\right) .
$$

The $\mathbf{x}_{k}$ integrations can then be performed which gives $\mathbf{x}_{j}$ $=\mathbf{x}_{0}+\epsilon \sum_{i=1}^{j} \mathbf{v}_{j}$, or in the continuous notation the trajectory $\mathbf{x}(\mathbf{t})=\mathbf{x}_{a}+\int_{0}^{t} d t^{\prime} \mathbf{v}\left(t^{\prime}\right)$. However, one $\delta$ function remains and we obtain

$$
\begin{aligned}
U\left(\mathbf{x}_{b}, t_{b} ; \mathbf{x}_{a}, t_{a}\right) & =\lim _{N \rightarrow \infty}\left(\frac{\epsilon m}{2 \pi i}\right)^{3 N / 2} \int d^{3} v_{1} \cdots d^{3} v_{N} \delta^{(3)}\left(\mathbf{x}_{b}-\mathbf{x}_{a}-\epsilon \sum_{j=1}^{N} \mathbf{v}_{j}\right) \exp \left\{i \epsilon \sum_{j=1}^{N}\left[\frac{m}{2} \mathbf{v}_{j}^{2}-V\left(\mathbf{x}_{j}=\mathbf{x}_{a}+\epsilon \sum_{i=1}^{j} \mathbf{v}_{i}\right)\right]\right\} \\
& \equiv \mathcal{N}^{3}\left(t_{a}, t_{b}\right) \int \mathcal{D}^{3} v \delta^{(3)}\left(\mathbf{x}_{b}-\mathbf{x}_{a}-\int_{t_{a}}^{t_{b}} d t \mathbf{v}(t)\right) \exp \left\{i \int_{t_{a}}^{t_{b}} d t\left[\frac{m}{2} \mathbf{v}^{2}(t)-V(\mathbf{x}(t))\right]\right\}
\end{aligned}
$$

Here the "measure" is given by $\mathcal{D}^{3} v=\Pi_{k}^{N} d^{3} v_{k}$ and the normalization factor

$$
\mathcal{N}\left(t_{a}, t_{b}\right):=\left(\int \mathcal{D} v \exp \left[i \int_{t_{a}}^{t_{b}} d t \frac{m}{2} v^{2}(t)\right]\right)^{-1}
$$

ensures that the Gaussian integral gives unity as is evident from the discrete form. Note that the functional integral over $\mathbf{v}$ does not require any boundary conditions which are all contained in the remaining $\delta$ function. A more symmetrical form for the argument of the potential is obtained by writing

$$
\mathbf{x}(t)=\frac{\mathbf{x}_{a}+\mathbf{x}_{b}}{2}+\frac{1}{2} \int_{t_{a}}^{t_{b}} d t^{\prime} \operatorname{sgn}\left(t-t^{\prime}\right) \mathbf{v}\left(t^{\prime}\right)
$$

where $\operatorname{sgn}(x)=x /|x|$ is the sign function. We have $\dot{\mathbf{x}}(t)=\mathbf{v}(t)$ and the boundary conditions for the paths are fulfilled due to the $\delta$ function in Eq. (4).

We now write Eq. (2) as

$$
S_{i \rightarrow f}=\lim _{T \rightarrow \infty} e^{i\left(E_{i}+E_{f}\right) T} \int d^{3} x d^{3} y e^{-i \mathbf{k}_{f} \mathbf{x}} U(\mathbf{x}, T ; \mathbf{y},-T) e^{i \mathbf{k}_{i} \cdot \mathbf{y}}
$$

and insert the representation (4). Using the coordinates $\mathbf{r}$ $=(\mathbf{x}+\mathbf{y}) / 2, \mathbf{s}=\mathbf{x}-\mathbf{y}$ we then obtain 


$$
\begin{aligned}
S_{i \rightarrow f}= & \lim _{T \rightarrow \infty} e^{i\left(E_{i}+E_{f}\right) T} \int d^{3} r e^{-i \mathbf{q} \cdot \mathbf{r}} \mathcal{N}^{3}(T,-T) \\
& \times \int \mathcal{D}^{3} v \exp \left\{i \int_{-T}^{+T} d t\left[\frac{m}{2} \mathbf{v}^{2}(t)-\mathbf{K} \cdot \mathbf{v}(t)\right]\right\} \\
& \times \exp \left\{-i \int_{-T}^{+T} d t V\left(\mathbf{r}+\mathbf{x}_{v}(t)\right)\right\},
\end{aligned}
$$

since the relative coordinate $\mathbf{s}$ is fixed by the $\delta$ function in Eq. (4). Here we have defined the momentum transfer and the mean momentum by

$$
\mathbf{q}=\mathbf{k}_{f}-\mathbf{k}_{i}, \quad \mathbf{K}=\frac{1}{2}\left(\mathbf{k}_{i}+\mathbf{k}_{f}\right) .
$$

Furthermore,

$$
\mathbf{x}_{v}(t)=\frac{1}{2} \int_{-T}^{+T} d t^{\prime} \operatorname{sgn}\left(t-t^{\prime}\right) \mathbf{v}\left(t^{\prime}\right)
$$

where the subscript denotes the dependence on the variable over which one is integrating functionally [24]. The shift $\mathbf{v}(t) \rightarrow \mathbf{v}(t)+\mathbf{K} / m$ eliminates the linear term in the exponent of the functional integral (8). Since

$$
\int_{-T}^{+T} d t^{\prime} \operatorname{sgn}\left(t-t^{\prime}\right)=2 t \quad \text { for } t \in[-T,+T]
$$

and

$$
\begin{aligned}
E_{i}+E_{f}-\frac{\mathbf{K}^{2}}{m} & =\frac{2 \mathbf{k}_{i}^{2}+2 \mathbf{k}_{f}^{2}-\left(\mathbf{k}_{i}+\mathbf{k}_{f}\right)^{2}}{4 m} \\
& =\frac{\left(\mathbf{k}_{i}-\mathbf{k}_{f}\right)^{2}}{4 m}=\frac{\mathbf{q}^{2}}{4 m}
\end{aligned}
$$

we obtain

$$
\begin{aligned}
S_{i \rightarrow f}= & \lim _{T \rightarrow \infty} \exp \left(i \frac{\mathbf{q}^{2}}{4 m} T\right) \\
& \times \int d^{3} r e^{-i \mathbf{q} \cdot \mathbf{r}} \mathcal{N}^{3}(T,-T) \int \mathcal{D}^{3} v \exp \left[i \int_{-T}^{+T} d t \frac{m}{2} \mathbf{v}^{2}(t)\right] \\
& \times \exp \left[-i \int_{-T}^{+T} d t V\left(\mathbf{r}+\frac{\mathbf{K}}{m} t+\mathbf{x}_{v}(t)\right)\right]
\end{aligned}
$$

Note that Eq. (12) is valid without energy conservation which has not been imposed (derived) yet. With no interaction we obtain

$$
S_{i \rightarrow f}^{(0)}=\lim _{T \rightarrow \infty} \exp \left(i \frac{\mathbf{q}^{2}}{4 m} T\right)(2 \pi)^{3} \delta^{(3)}(\mathbf{q})=(2 \pi)^{3} \delta^{(3)}\left(\mathbf{k}_{i}-\mathbf{k}_{f}\right)
$$

and therefore we will consider

$$
(S-1)_{i \rightarrow f}=\lim _{T \rightarrow \infty} \exp \left(i \frac{\mathbf{q}^{2}}{4 m} T\right) \int d^{3} r e^{-i \mathbf{q} \cdot \mathbf{r}} \mathcal{N}^{3}(T,-T) \int \mathcal{D}^{3} v \exp \left[i \int_{-T}^{+T} d t \frac{m}{2} \mathbf{v}^{2}(t)\right]\left\{\exp \left[-i \int_{-T}^{+T} d t V\left(\mathbf{r}+\frac{\mathbf{K}}{m} t+\mathbf{x}_{v}(t)\right)\right]-1\right\}
$$

in the following. Since the potential vanishes at infinity Eq. (15) is a well-defined integral.

\section{ASYMPTOTIC TIMES: ELIMINATION OF DANGEROUS PHASES}

The path integral representation (15) is exact but suffers in the present formulation from the explicit appearance of a "dangerous phase" $\mathbf{q}^{2} T /(4 m)$ proportional to $T$, in the first exponential of Eq. (15). It can be checked, of course, that this phase cancels in each order of perturbation theory so that the limit $T \rightarrow \infty$ can indeed be performed but one would like to have a formulation where this phase does not appear at all. This can be achieved by recognizing that each power of $\mathbf{q}^{2}$ arises from applying the three-dimensional Laplacian $-\Delta$ to the factor $\exp (-i \mathbf{q} \cdot \mathbf{r})$ in the integral over $\mathbf{r}$. An integration by parts then lets it act on the potential term [25]. In order to reduce it to a shift operator one may "undo the square," for example by a three-dimensional path integral

$$
\begin{aligned}
\exp (- & \left.\frac{i}{4 m} T \Delta\right)=\mathcal{N}^{* 3}(T,-T) \int \mathcal{D}^{3} w \\
& \times \exp \left[-i \int_{-T}^{+T} d t \frac{m}{2} \mathbf{w}^{2}(t) \pm \int_{-T}^{+T} d t \frac{1}{2} f(t) \mathbf{w}(t) \cdot \nabla\right]
\end{aligned}
$$

Here $f(t)$ should fulfill

$$
\int_{-T}^{+T} d t f^{2}(t)=2 T
$$

and the normalization again ensures that the pure Gaussian integral gives unity.

Note that the sign of the quadratic term in the exponent necessarily is reversed if one wants to have a real shift operator whereas the linear term can have any sign. Real arguments of the potential are mandatory if an analytic continuation of the potential into the complex plane is to be avoided. Such a procedure would depend on the specific analytic 
properties of the potential and would have to be considered on a case-by-case basis. We will call $\mathbf{w}(t)$ an "antivelocity" and choose the negative sign in the linear term for convenience. Also we will take

$$
f(t)=\operatorname{sgn}(-t)
$$

so that the shift operator simply becomes

$$
\exp \left[-\frac{1}{2} \int_{-T}^{+T} d t \operatorname{sgn}(-t) \mathbf{w}(t) \cdot \nabla\right]=\exp \left[-\mathbf{x}_{w}(0) \cdot \nabla\right]
$$

and the antivelocity degrees of freedom are as close to the velocity ones as possible. Then we obtain the following pathintegral representation for the $S$ matrix:

$$
\begin{aligned}
(S-1)_{i \rightarrow f} & =\lim _{T \rightarrow \infty} \int d^{3} r e^{-i \mathbf{q} \cdot \mathbf{r}}|\mathcal{N}(T,-T)|^{6} \int \mathcal{D}^{3} v \mathcal{D}^{3} w \\
& \times \exp \left[i \int_{-T}^{+T} d t \frac{m}{2}\left[\mathbf{v}^{2}(t)-\mathbf{w}^{2}(t)\right]\right] \\
& \times\left\{\exp \left[-i \int_{-T}^{+T} d t V\left(\mathbf{r}+\frac{\mathbf{K}}{m} t+\mathbf{x}_{v}(t)-\mathbf{x}_{w}(0)\right)\right]-1\right\} .
\end{aligned}
$$

There is an interesting analogy with the Lee-Wick approach to quantum electrodynamics where also fields with a wrongsign kinetic term are introduced [26] to remove all infinities. These "phantom" degrees of freedom are often described in an indefinite inner product space [27]. In our case, however, they are not conjectured but necessarily appear when eliminating the asymptotically diverging phase $\mathbf{q}^{2} T /(4 m)$ in the $S$ matrix.

At first sight the present approach to remove the infinite phase looks as if the phase space path integral used in Ref. [18] has come back through the backdoor in disguise of a functional integration over velocities and antivelocities. However, since the argument of the potential in Eq. (20) only depends on the fixed quantity $\mathbf{x}_{w}(0)$, the path integral over the antivelocity is not a full functional integral but could be replaced by an ordinary one. Such a representation corresponds to using

$$
\begin{gathered}
\exp \left(-\frac{i}{4 m} T \Delta\right)=\left[\int d^{3} w \exp \left(-i m T \mathbf{w}^{2}\right)\right]^{-1} \\
\times \int d^{3} w \exp \left[-i m T \mathbf{w}^{2} \pm T \mathbf{w} \cdot \nabla\right],
\end{gathered}
$$

i.e., a constant antivelocity $\mathbf{w}$, instead of Eq. (16). This may offer definite advantages in all cases where an additional functional integration would be costly as in attempts to evaluate the real-time path integral numerically. However, compared to Eq. (16) it has the disadvantage that an explicit dependence on the time $T$ formally remains and that $\mathbf{v}, \mathbf{w}$ are treated differently. Therefore we will use the time-dependent antivelocity $\mathbf{w}(t)$ in the following applications.

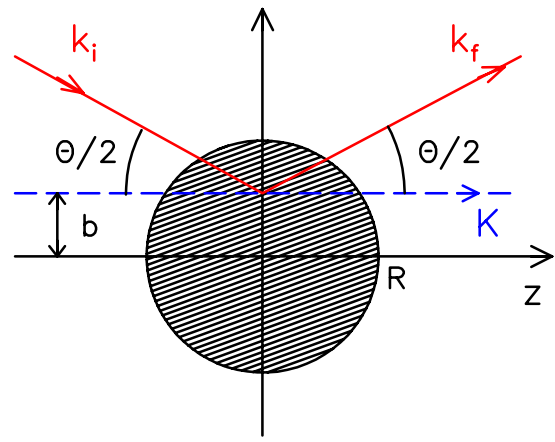

FIG. 1. (Color online). Scattering geometry for a potential of radius $R$, the impact parameter $b$, the ray made by the incoming and outgoing momenta $\mathbf{k}_{i, f}$, and the mean momentum $\mathbf{K}=\left(\mathbf{k}_{i}+\mathbf{k}_{f}\right) / 2$.

\section{FADDEEV-POPOV METHODS FOR THE $T$ MATRIX}

How to extract the $T$ matrix from the $S$ matrix? For weak interaction one can develop in powers of the potential and one finds that in each order an energy-conserving $\delta$ function can be factored out.

To achieve this without a perturbative expansion of the $S$ matrix one can use the trick which Faddeev and Popov (FP) have introduced in field theory for the quantization of nonAbelian gauge theories as was first proposed in Ref. [18]: We note that in the limit $T \rightarrow \infty$ the action in the path integral (20) is invariant under the transformation

$$
t=\bar{t}+\tau, \quad \mathbf{r}=\overline{\mathbf{r}}-\frac{\mathbf{K}}{m} \tau, \quad \mathbf{v}(t)=\overline{\mathbf{v}}(\bar{t}),
$$

since

$$
\begin{aligned}
\int_{-T}^{+T} d t V\left(\mathbf{r}+\frac{\mathbf{K}}{m} t+\mathbf{x}_{v}(t)-\mathbf{x}_{w}(0)\right) \\
=\int_{-T-\tau}^{T-\tau} d \bar{t} V\left(\overline{\mathbf{r}}+\frac{\mathbf{K}}{m} \bar{t}\right. \\
\left.\quad+\frac{1}{2} \int_{-T-\tau}^{T-\tau} \overline{d t^{\prime}} \overline{\mathbf{v}}\left(\overline{t^{\prime}}\right) \operatorname{sgn}\left(\bar{t}-\overline{t^{\prime}}\right)-\mathbf{x}_{w}(0)\right) .
\end{aligned}
$$

For finite $\tau$ and $T \rightarrow \infty$ one may expect that the change in the integral limits is of no relevance and therefore that the action remains invariant under the above transformation. Actually the limit $T \rightarrow \infty$ is nontrivial and needs a more rigorous investigation which is beyond the scope of the present investigation. Instead we will verify that our procedure is correct by checking that each term of the Born series emerges from our path integral representations. 
If the action is assumed to be invariant under the transformation (22) then it does not depend on the component of the vector $\mathbf{r}$ which is parallel to $\mathbf{K}$, leading to a singularity when integrating over that component. This singularity is just the energy-conserving $\delta$ function we are looking for. We can extract it by first fixing it and then integrating over all possible values: For example, we multiply the path integral (15) by the following factor:

$$
1=\frac{|\mathbf{K}|}{m} \int_{-\infty}^{+\infty} d \tau \delta\left(\hat{\mathbf{K}} \cdot\left[\mathbf{r}+\frac{\mathbf{K}}{m} \tau\right]+\lambda\right)
$$

where $\lambda$ is an arbitrary fixed ("gauge") parameter and $\hat{\mathbf{K}}$ $=\mathbf{K} /|\mathbf{K}|$ the unit vector in the $\mathbf{K}$ direction.

We now perform the transformation (22) in the path integral and obtain

$$
\begin{aligned}
(S-1)_{i \rightarrow f}= & \frac{|\mathbf{K}|}{m} \lim _{T \rightarrow \infty} \int_{-\infty}^{+\infty} d \tau \int d^{3} r \exp \left(-i \mathbf{q} \cdot \mathbf{r}+i \mathbf{q} \cdot \frac{\mathbf{K}}{m} \tau\right) \delta(\hat{\mathbf{K}} \cdot \mathbf{r}+\lambda)|\mathcal{N}(T,-T)|^{6} \int \mathcal{D}^{3} v \mathcal{D}^{3} w \\
& \times \exp \left\{i \int_{-T}^{+T} d t \frac{m}{2}\left[\mathbf{v}^{2}(t)-\mathbf{w}^{2}(t)\right]\right\}\left\{\exp \left[-i \int_{-T}^{+T} d t V\left(\mathbf{r}+\frac{\mathbf{K}}{m} t+\mathbf{x}_{v}(t)-\mathbf{x}_{w}(0)\right)\right]-1\right\} .
\end{aligned}
$$

To simplify the nomenclature the original variables are used again. The only dependence on $\tau$ in the integrand now resides in the factor $\exp (-i \tau \mathbf{q} \cdot \mathbf{K} / m)$ and thus the integration over it produces the energy-conserving $\delta$ function [28]

$$
2 \pi \delta\left(\frac{\mathbf{q} \cdot \mathbf{K}}{m}\right)=2 \pi \delta\left(\frac{\mathbf{k}_{f}^{2}}{2 m}-\frac{\mathbf{k}_{i}^{2}}{2 m}\right) .
$$

In addition, after the transformation the longitudinal component of $\mathbf{r}$ is set to the value $-\lambda$. Noting that $q_{\|}=0$ we then obtain the following expression for the $T$ matrix:

$$
\begin{array}{rl}
T_{i \rightarrow f}^{(3-3)}=i & i \frac{K}{m} \int d^{2} b e^{-i \mathbf{q} \cdot \mathbf{b}}|\mathcal{N}|^{6} \int \mathcal{D}^{3} v \mathcal{D}^{3} w \\
& \times \exp \left[i \int_{-\infty}^{+\infty} d t \frac{m}{2}\left[\mathbf{v}^{2}(t)-\mathbf{w}^{2}(t)\right]\left\{e^{i \chi_{\mathbf{K}}(\mathbf{b}, \mathbf{v}, \mathbf{w})}-1\right\} .\right.
\end{array}
$$

Here we have taken the limit $T \rightarrow \infty$ and have written the corresponding Gaussian normalization factor as

$$
\mathcal{N}:=\mathcal{N}(+\infty,-\infty) .
$$

In Eq. (27) the phase $\chi_{\mathbf{K}}$ is defined as

$$
\begin{aligned}
\chi_{\mathbf{K}}(\mathbf{b}, \mathbf{v}, \mathbf{w})= & -\int_{-\infty}^{+\infty} d t V\left(\mathbf{b}+\frac{\mathbf{K}}{m} t+\mathbf{x}_{v}(t)-\mathbf{x}_{w}(0)-\lambda \hat{\mathbf{K}}\right) \\
& =:-\int_{-\infty}^{+\infty} d t V\left(\xi_{\mathbf{K}}(t)\right)
\end{aligned}
$$

while $\mathbf{b} \equiv \mathbf{r}_{\perp}$ denotes the transverse component of the vector $\mathbf{r}$ (the impact parameter). With $\theta$ being the scattering angle, we have

$$
q \equiv|\mathbf{q}|=2 k \sin \left(\frac{\theta}{2}\right), \quad K \equiv|\mathbf{K}|=k \cos \left(\frac{\theta}{2}\right) .
$$

Writing $\lambda=K t_{0} / m$ we see that the "gauge parameter" can be traded for an arbitrary time $t_{0}$ in the reference path $\mathbf{b}+\mathbf{K}(t$ $\left.-t_{0}\right) / m$. We expect that $\lambda=0$, i.e., $t_{0}=0$ is the most symmetric choice (see below).

As an exact path-integral representation of the $T$ matrix (27) is one of the major results of this paper. The superscript "3-3" indicates that in addition to the three-dimensional velocity variable a three-dimensional antivelocity is used to cancel divergent phases in the limit of asymptotic times. Using Eq. (4) backwards it is also possible to write the result as an ordinary path integral over paths $\mathbf{x}(t), \mathbf{y}(t)$ instead of velocities $\mathbf{v}(t), \mathbf{w}(t)$. These paths have to fulfill boundary conditions $\mathbf{x}( \pm T)= \pm \mathbf{x}_{0} / 2, \mathbf{y}( \pm T)= \pm \mathbf{y}_{0} / 2$ and one has to integrate over $\mathbf{x}_{0}, \mathbf{y}_{0}$ at the end. However, this brings neither simplifications nor new insights and so we will not pursue it further. Instead we will show in the next section that one can obtain the desired cancellation of divergent phases with a one-dimensional (longitudinal) antivelocity only.

\section{RAY REPRESENTATION}

The representation (27) can be simplified by a simultaneous shift of the impact parameter and the velocities

$$
\mathbf{v}(t)=\frac{\mathbf{q}}{2 m} \operatorname{sgn}(t)+\mathbf{v}^{\prime}(t), \quad \mathbf{w}(t)=\frac{\mathbf{q}}{2 m} \operatorname{sgn}(t)+\mathbf{w}^{\prime}(t),
$$

$$
\mathbf{b}=\mathbf{b}^{\prime}-\mathbf{x}_{v^{\prime} \perp}(0)+\mathbf{x}_{w^{\prime} \perp}(0) .
$$

This transformation is suggested by a stationary phase approximation to Eq. (27)

$$
\frac{\delta}{\delta \mathbf{v}(s)} \int_{-T}^{+T} d t\left[\frac{m}{2} \mathbf{v}^{2}(t)-V\left(\xi_{\mathbf{K}}(t)\right)\right] \stackrel{!}{=} 0,
$$

which gives for the stationary values of velocity and impact parameter

$$
m \mathbf{v}^{\text {stat }}(s)=\int_{-T}^{+T} d t \nabla V\left(\xi_{\mathbf{K}}(t)\right) \frac{1}{2} \operatorname{sgn}(t-s),
$$




$$
\begin{aligned}
m \mathbf{w}^{\text {stat }}(s) & =\int_{-T}^{+T} d t \nabla V\left(\boldsymbol{\xi}_{\mathbf{K}}(t)\right) \frac{1}{2} \operatorname{sgn}(-s), \\
\mathbf{q} & =-\int_{-T}^{+T} d t \nabla_{b} V\left(\boldsymbol{\xi}_{\mathbf{K}}(t)\right) .
\end{aligned}
$$

We thus find $\mathbf{w}_{\perp}^{\text {stat }}(s)=\mathbf{q} /(2 m) \operatorname{sgn}(s)$ and for small scattering times [29] $t$ or asymptotic external times $s$ also

$$
\mathbf{v}_{\perp}^{\text {stat }}(s) \approx \frac{\mathbf{q}}{2 m} \operatorname{sgn}(s),
$$

which suggests the shift (31). However, doing so introduces additional terms in the exponent since

$$
\begin{aligned}
\frac{m}{2} \int_{-T}^{+T} d t \mathbf{v}^{2}(t)= & \frac{m}{2} \int_{-T}^{+T} d t \mathbf{v}^{\prime 2}(t) \\
& +\mathbf{q} \cdot \frac{1}{2} \int_{-T}^{+T} d t \operatorname{sgn}(t) \mathbf{v}^{\prime}(t)+\frac{\mathbf{q}^{2}}{4 m} T \\
= & \frac{m}{2} \int_{-T}^{+T} d t \mathbf{v}^{\prime 2}(t)-\mathbf{q} \cdot \mathbf{x}_{v^{\prime} \perp}(0)+\frac{\mathbf{q}^{2}}{4 m} T
\end{aligned}
$$

Similarly

$$
\frac{m}{2} \int_{-T}^{+T} d t \mathbf{w}^{2}(t)=\int_{-T}^{+T} d t \mathbf{w}^{\prime 2}(t)-\mathbf{q} \cdot \mathbf{x}_{w^{\prime} \perp}(0)+\frac{\mathbf{q}^{2}}{4 m} T,
$$

so that

$$
\begin{aligned}
\frac{m}{2} \int_{-\infty}^{+\infty} d t\left[\mathbf{v}^{2}(t)-\mathbf{w}^{2}(t)\right]= & \int_{-\infty}^{+\infty}\left[\mathbf{v}^{\prime 2}(t)-\mathbf{w}^{\prime 2}(t)\right] \\
& +\mathbf{q} \cdot\left[\mathbf{x}_{w^{\prime} \perp}(0)-\mathbf{x}_{v^{\prime} \perp}(0)\right]
\end{aligned}
$$

is independent of the time $T$ used for regularization. But finite terms remain which are then canceled by the shift (32) of the impact parameter. Note that only the transverse component of $\mathbf{x}_{v^{\prime}}$ and $\mathbf{x}_{w^{\prime}}$ can appear in Eq. (32) since the impact parameter necessarily is a two-dimensional vector. This asymmetry between perpendicular and parallel components can be traced back to the constraint (24) and will persist in the following formulas. Using the relation [30]

$$
\begin{array}{r}
\int_{-T}^{+T} d s \operatorname{sgn}(s-t) \operatorname{sgn}\left(s-t^{\prime}\right)=2\left[T-\left|t-t^{\prime}\right|\right], \\
t, t^{\prime} \in[-T,+T]
\end{array}
$$

we find from Eqs. (10) and (31) that

$$
\begin{aligned}
& \mathbf{x}_{v}(t)=\frac{\mathbf{q}}{2 m}[|t|-T]+\mathbf{x}_{v^{\prime}}(t), \\
& \mathbf{x}_{w}(0)=\frac{\mathbf{q}}{2 m}(-T)+\mathbf{x}_{w^{\prime}}(0) .
\end{aligned}
$$

Therefore the argument of the potential term also becomes (formally) $T$ independent

$$
\begin{aligned}
\boldsymbol{\xi}_{\mathbf{K}}(t) \rightarrow \boldsymbol{\xi}_{\text {ray }}(t)= & \mathbf{b}^{\prime}+\frac{\mathbf{p}_{\text {ray }}(t)}{m} t-\lambda \hat{\mathbf{K}}+\mathbf{x}_{v^{\prime}}(t)-\mathbf{x}_{v^{\prime} \perp}(0) \\
& -x_{w^{\prime} \|}(0) \hat{\mathbf{K}}
\end{aligned}
$$

Here

$$
\mathbf{p}_{\text {ray }}(t)=\mathbf{K}+\frac{\mathbf{q}}{2} \operatorname{sgn}(t)=\mathbf{k}_{i} \Theta(-t)+\mathbf{k}_{f} \Theta(t)
$$

is the new momentum along which the particle mainly travels: for $t<0$ it is the initial momentum and for $t>0$ it is the final momentum. This is also what one expects intuitively at high energies and is depicted in Fig. 1. Note that the magnitude of $\mathbf{p}_{\text {ray }}(t)$ is $k$ for all $t$ and therefore the velocity of the high-energy particle along the "rays" remains the asymptotic $k / m$ instead of the unnatural $K / m=k \cos (\theta / 2) / m$.

After the shifting of arguments the integrand does not depend on $\mathbf{x}_{w \perp}(0)$, i.e., $\mathbf{w}_{\perp}(t)$ anymore. Therefore the integration over the perpendicular components of $\mathbf{w}(t)$ can be performed trivially cancelling the corresponding Gaussian normalization constants. Choosing $\lambda=0$, omitting the prime for the shifted variables and writing $w$ for $w_{\|}$the new path integral representation now reads

$$
\begin{aligned}
T_{i \rightarrow f}^{(3-1)} & =i \frac{K}{m} \int d^{2} b e^{-i \mathbf{q} \cdot \mathbf{b}} \mathcal{N}^{3} \mathcal{N}^{*} \int \mathcal{D}^{3} v \mathcal{D} w \\
& \times \exp \left[i \int_{-\infty}^{+\infty} d t \frac{m}{2}\left(\mathbf{v}^{2}(t)-w^{2}(t)\right)\right] \\
& \times\left\{e^{i \chi_{\text {ray }}(\mathbf{b}, \mathbf{v}, w)}-1\right\}
\end{aligned}
$$

with only one (longitudinal) antivelocity which is indicated by the superscript " $3-1$ ". The phase is given by

$$
\begin{aligned}
\chi_{\text {ray }}(\mathbf{b}, \mathbf{v}, w)= & -\int_{-\infty}^{+\infty} d t V\left(\mathbf{b}+\frac{\mathbf{p}_{\text {ray }}(t)}{m} t+\mathbf{x}_{v}(t)-\mathbf{x}_{v \perp}(0)\right. \\
& \left.-x_{w}(0)\right)
\end{aligned}
$$

Note that both path integral representations of the $T$ matrix are not impact parameter representations in the strict sense since both the phases $\chi$ and the factor $K=k \cos (\theta / 2)$ carry an angle dependence whereas in an exact impact parameter representation of the $T$ matrix this dependence would only reside in the factor $\exp (-i \mathbf{q} \cdot \mathbf{b})$ [31]. As a consequence, unitarity of the $S$ matrix, i.e., validity of the optical theorem is not immediately evident although these are exact path integral representations.

\section{A. Microreversibility}

It is worthwhile to explore how microreversibility (time reversal) of the $T$ matrix [32] is realized in the present path integral approach. This is the invariance under the exchange 


$$
\mathbf{k}_{i} \rightarrow-\mathbf{k}_{f}, \quad \mathbf{k}_{f} \rightarrow-\mathbf{k}_{i}
$$

i.e.,

$$
\mathbf{q} \rightarrow \mathbf{q}, \quad \mathbf{K} \rightarrow-\mathbf{K} .
$$

We first note that the gauge parameter $\lambda$ has to vanish since it multiplies the odd vector $\hat{\mathbf{K}}$ in the argument $\boldsymbol{\xi}_{\mathbf{K}}(t)$ of the phase

$$
\lambda=0 \text {. }
$$

This is also evident from the FP constraint (24), where the $\operatorname{argument}$ of the $\delta$ function would have different parity upon time-reversal or simply by considering $\lambda$ as an arbitrary time scale $t_{0}$ for the longitudinal motion which would destroy the time-symmetry between initial and final states.

However, microreversibility does not constrain the dynamical variable $\mathbf{v}(t)$. Let us discuss that for the case of an one-dimensional antivelocity with the phase $\chi_{\text {ray }}(\mathbf{b}, \mathbf{v}, w)$ given in Eq. (45): the impact parameter $\mathbf{b}$ is unaffected, but the reference path obviously changes under the transformations (47):

$$
\frac{\mathbf{p}_{\text {ray }}(t)}{m} t \rightarrow-\frac{\mathbf{K}}{m} t+\frac{\mathbf{q}}{2 m}|t| .
$$

This can be compensated [33] by changing the integration variable $t \rightarrow-t$ :

$$
\chi_{\text {ray }}(\mathbf{b}, \mathbf{v}, w) \rightarrow-\int_{-\infty}^{+\infty} d t V\left[\boldsymbol{\xi}_{\text {ray }}(-t)\right]
$$

where

$$
\boldsymbol{\xi}_{\text {ray }}(-t)=\mathbf{b}+\frac{\mathbf{K}}{m} t+\frac{\mathbf{q}}{2 m}|t|+\mathbf{x}_{v}(-t)-\mathbf{x}_{v \perp}(0)+x_{w}(0) \hat{\mathbf{K}}
$$

and

$$
\begin{aligned}
\mathbf{x}_{v}(-t) & =\frac{1}{2} \int_{-\infty}^{+\infty} d t^{\prime} \operatorname{sgn}\left(-t-t^{\prime}\right) \mathbf{v}\left(t^{\prime}\right) \\
& =\frac{1}{2} \int_{-\infty}^{+\infty} d t^{\prime} \operatorname{sgn}\left(t-t^{\prime}\right)(-) \mathbf{v}\left(-t^{\prime}\right) .
\end{aligned}
$$

Decomposing the variable $\mathbf{v}(t)$ into even and odd components

$$
\mathbf{v}(t)=\mathbf{v}_{+}(t)+\mathbf{v}_{-}(t) \quad \text { with } \mathbf{v}_{ \pm}(-t)= \pm \mathbf{v}_{ \pm}(t)
$$

one sees that the kinetic term is quadratic in both components

$$
\frac{m}{2} \int_{-\infty}^{+\infty} d t \mathbf{v}^{2}(t)=\frac{m}{2} \int_{-\infty}^{+\infty} d t\left[\mathbf{v}_{+}^{2}(t)+\mathbf{v}_{-}^{2}(t)\right]
$$

This allows us to transform

$$
\mathbf{x}_{v}(-t)=\frac{1}{2} \int_{-\infty}^{+\infty} d t^{\prime} \operatorname{sgn}\left(t-t^{\prime}\right)(-)\left[\mathbf{v}_{+}\left(t^{\prime}\right)-\mathbf{v}_{-}\left(t^{\prime}\right)\right]
$$

into $\mathbf{x}_{v}(t)$ by a simple change of integration variables

$$
\mathbf{v}_{+}(t) \rightarrow-\mathbf{v}_{+}(t)
$$

in the velocity path integral (leaving $\mathbf{v}_{-}$unchanged) and demonstrates invariance of the phase $\chi_{\text {ray }}$ and of the whole $T$ matrix. Of course, the subtraction terms

$$
\begin{aligned}
\mathbf{x}_{v \perp}(0) & =\frac{1}{2} \int_{-\infty}^{+\infty} d t^{\prime} \operatorname{sgn}\left(-t^{\prime}\right) \mathbf{v}_{\perp}\left(t^{\prime}\right), \\
x_{w}(0) & =\frac{1}{2} \int_{-\infty}^{+\infty} d t^{\prime} \operatorname{sgn}\left(-t^{\prime}\right) w\left(t^{\prime}\right)
\end{aligned}
$$

depend only on the time-odd components. For the case of a three-dimensional antivelocity the arguments are even simpler but completely analogous.

\section{B. Tests}

As a test for the correct treatment of the various limits and shifts which we have performed, the Born series should be obtained from the path integral representations $T^{(3-3)}$ and $T^{(3-1)}$. Here we only consider the first Born approximation while terms of arbitrary order are evaluated in Appendix A. The first-order $T$ matrix is simply obtained by expanding the corresponding phase to linear order and Fourier transforming the potential

$$
\begin{aligned}
T_{i \rightarrow f}^{(3-3) \text { Born }}= & \frac{K}{m} \int d^{2} b e^{-i \mathbf{q} \cdot \mathbf{b}} \int \frac{d^{3} p}{(2 \pi)^{3}} \tilde{V}(\mathbf{p}) \mid \mathcal{N}^{6} \\
& \times \int \mathcal{D}^{3} v \mathcal{D}^{3} w \exp \left[\frac{i m}{2} \int_{-\infty}^{+\infty} d t\left[\mathbf{v}^{2}(t)-\mathbf{w}^{2}(t)\right]\right] \\
& \times \int_{-\infty}^{+\infty} d s \exp \left\{i \mathbf{p} \cdot\left[\mathbf{b}+\frac{\mathbf{K}}{m} s+\mathbf{x}_{v}(s)-\mathbf{x}_{w}(0)\right]\right\} .
\end{aligned}
$$

The functional integrals here are simple Gaussian ones of the form

$$
\begin{aligned}
G^{(d)} & :=\mathcal{N}^{d} \int \mathcal{D}^{d} v \exp \left\{i \int_{-T}^{+T} d t\left[\frac{m}{2} \mathbf{v}^{2}(t)+\mathbf{g}(t) \cdot \mathbf{v}(t)\right]\right\} \\
& =\exp \left[-i \int_{-T}^{+T} d t \frac{\mathbf{g}^{2}(t)}{2 m}\right]
\end{aligned}
$$

and we let the time $T$ go to infinity only at the end of the calculation. From the relation (10) we read off $\mathbf{g}_{v}(t)$ $=\mathbf{p} \operatorname{sgn}(s-t) / 2$ for the $\mathbf{v}$ integration and $\mathbf{g}_{w}(t)=\mathbf{p} \operatorname{sgn}(-t) / 2$ for the $\mathbf{w}$ integration. Thus

$$
\begin{aligned}
G_{v}^{(d=3)} G_{w}^{(d=3) *} & =\exp \left\{-\frac{i \mathbf{p}^{2}}{8 m} \int_{-T}^{+T} d t\left[\operatorname{sgn}^{2}(s-t)-\operatorname{sgn}^{2}(-t)\right]\right\} \\
& =\exp \left\{-i \frac{\mathbf{p}^{2}}{8 m}(2 T-2 T)\right\}=1
\end{aligned}
$$

and 


$$
T_{i \rightarrow f}^{(3-3) \text { Born }}=\frac{K}{m} \int d^{2} b e^{-i \mathbf{q} \cdot \mathbf{b}} \int \frac{d^{3} p}{(2 \pi)^{3}} \tilde{V}(\mathbf{p}) \lim _{T \rightarrow \infty} \int_{T}^{+T} d s \exp \left(i \mathbf{p} \cdot \mathbf{b}+i \mathbf{p} \cdot \frac{\mathbf{K}}{m} s\right)=\int d^{3} p \tilde{V}(\mathbf{p}) \delta^{(2)}\left(\mathbf{p}{ }_{\perp}-\mathbf{q}\right) \frac{K}{m} \delta\left(\frac{K}{m} p_{\|}\right)=\tilde{V}(\mathbf{q}, 0) \equiv \tilde{V}(\mathbf{q})
$$

as expected.

Although the ray representation (44) was derived by a simple shift of integration variables from Eq. (27) and therefore did not involve any additional large- $T$ limits it is instructive to derive the first Born approximation explicitly in this case too. We have

$$
\begin{aligned}
T_{i \rightarrow f}^{(3-1) \text { Born }}= & \frac{K}{m} \int d^{2} b e^{-i \mathbf{q} \cdot \mathbf{b}} \int \frac{d^{3} p}{(2 \pi)^{3}} \tilde{V}(\mathbf{p}) \int \mathcal{D}^{3} v \mathcal{D} w \exp \left[i \frac{m}{2} \int_{-\infty}^{+\infty} d t\left[\mathbf{v}^{2}(t)-w^{2}(t)\right]\right] \\
& \times \int_{-\infty}^{+\infty} d s \exp \left\{i \mathbf{p} \cdot\left[\mathbf{b}+\frac{\mathbf{p}_{\text {ray }}(s)}{m} s+\mathbf{x}_{v}(s)-\mathbf{x}_{v \perp}(0)-x_{w}(0) \hat{K}\right]\right\} .
\end{aligned}
$$

From the master path integral (59) we obtain

$$
\begin{aligned}
G_{v}^{(d=3)} G_{w}^{(d=1) *}= & \exp \left\{-\frac{i}{8 m} \int_{-T}^{+T} d t\left\{p_{\perp}^{2}[\operatorname{sgn}(s-t)-\operatorname{sgn}(-t)]^{2}\right.\right. \\
& \left.\left.+p_{\|}^{2}\left[\operatorname{sgn}^{2}(s-t)-\operatorname{sgn}^{2}(-t)\right]\right\}\right\}
\end{aligned}
$$

corresponding to perpendicular and parallel path integration over the velocities $\mathbf{v}, w$. The longitudinal component of the momentum $\mathbf{p}$ is completely cancelled by the contribution from the antivelocity $w$ but now a term remains in the exponent which is proportional to $\mathbf{p}_{\perp}^{2}$. Performing the $t$ integration by means of Eq. (39) all $T$ dependence cancels and we obtain

$$
G_{v}^{(d=3)} G_{w}^{(d=1) *}=\exp \left\{-\frac{i}{2 m} \mathbf{p}_{\perp}^{2}|s|\right\}
$$

Using the explicit form (43) of the momentum $\mathbf{p}_{\text {ray }}(s)$ it follows that

$$
\begin{aligned}
T_{i \rightarrow f}^{(3-1) \text { Born }}= & \frac{K}{m} \int d^{2} b e^{-i \mathbf{q} \cdot \mathbf{b}} \int \frac{d^{3} p}{(2 \pi)^{3}} \tilde{V}(\mathbf{p}) \\
& \times \int_{-\infty}^{+\infty} d s \exp \left(i \mathbf{p} \cdot \mathbf{b}+i \mathbf{p} \cdot \frac{\mathbf{K}}{m} s+i \mathbf{p} \cdot \frac{\mathbf{q}}{2 m}|s|\right. \\
& \left.-\frac{i}{2 m} \mathbf{p}_{\perp}^{2}|s|\right) .
\end{aligned}
$$

The $\mathbf{b}$ integration leads to $\mathbf{p}_{\perp}=\mathbf{q}$ and therefore the leftover term from the $\mathbf{v}_{\perp}$ integration is taken away by the contribution from the modified reference path. Thus we obtain again the correct first-order result (61).

In Appendix A we show how to obtain the complete Born series from these two path integral representations. This demonstrates that they are completely equivalent to the standard (time-independent) scattering theory and can be utilized without doubt.

\section{HIGH-ENERGY EXPANSIONS}

The path integral representations (27) and (44) for the $T$ matrix are the natural starting points for high-energy approximations. Under these kinematical conditions one expects that the particle essentially moves along straight lines with a constant velocity and that the functional integral over velocity and antivelocity only describes the fluctuations around this trajectory.

\section{A. Eikonal expansion}

Taking Eq. (27) (where the particle travels along the mean momentum $\mathbf{K}$ ) as reference one indeed finds that this is the case. By setting

$$
t=\frac{m}{K} z, \quad \mathbf{v}(t)=\frac{\sqrt{K}}{m} \overline{\mathbf{v}}(z), \quad \mathbf{w}(t)=\frac{\sqrt{K}}{m} \overline{\mathbf{w}}(z)
$$

it is seen that the path integral (27) takes the form

$$
\begin{aligned}
T_{i \rightarrow f}^{(3-3)}= & i \frac{K}{m} \int d^{2} b e^{-i \mathbf{q} \cdot \mathbf{b}}|\overline{\mathcal{N}}|^{6} \int \mathcal{D}^{3} \bar{v} \mathcal{D}^{3} \bar{w} \\
& \times \exp \left\{\frac{i}{2} \int_{-\infty}^{+\infty} d z\left[\overline{\mathbf{v}}^{2}(z)-\overline{\mathbf{w}}^{2}(z)\right]\right\} \\
& \times\left\{\operatorname { e x p } \left[-i \frac{m}{K} \int_{-\infty}^{+\infty} d z V\left(\mathbf{b}+\hat{\mathbf{K}} z+\frac{1}{\sqrt{K}}\left[\mathbf{x}_{\bar{v}}(z)\right.\right.\right.\right. \\
& \left.\left.\left.\left.-\mathbf{x}_{\bar{w}}(0)\right]\right)\right]-1\right\} .
\end{aligned}
$$

In many applications (e.g., in atomic physics) the energy of the incoming particle is not large compared to its rest mass. Therefore we consider $m / K$ not as small but as fixed in the following. Equation (67) shows that this factor just multiplies the potential but-irrespective of its magnitude - a systematic expansion in inverse powers of $K$ of the $T$ matrix is possible for fixed momentum transfer (or scattering angle). This is achieved just by expanding the phase simultaneously in powers of $\mathbf{v}(t), \mathbf{w}(t)$ and performing the functional integral 
term by term: At high energy the fluctuations around the straight-line trajectory are indeed small. Of course, the convergence will depend on size and smoothness of the potential as higher and higher derivatives of it will appear in the expansion. In addition, since $K=k \cos (\theta / 2)$ becomes smaller in backward direction the convergence of the expansion will deteriorate for larger scattering angles. A rough estimate of the validity of the expansion may be given by the requirement that the next order term of the Taylor expansion be small compared to the leading term

$$
\left|\frac{1}{\sqrt{K}} \nabla V \cdot \mathbf{x}_{\bar{v}}\right| \ll V
$$

Assuming that the velocity fluctuations are only relevant within the range $R$ of the potential one finds $\bar{v}=O(1 / \sqrt{R})$ and $x_{\bar{v}}=O(\sqrt{R})$ and thus

$$
K R \gg\left(R \frac{\nabla V}{V}\right)^{2} \simeq\left(\frac{R}{a}\right)^{2},
$$

where $a$ is the scale over which the potential changes appreciably.

Let us start with the lowest order term. Setting $\mathbf{v}=\mathbf{w}=0$ in the argument $\boldsymbol{\xi}_{\mathbf{K}}(t)$ of the potential immediately gives

$$
\begin{gathered}
T_{i \rightarrow f} \simeq T_{\mathrm{AI}}^{(0)}=i \frac{K}{m} \int d^{2} b e^{-i \mathbf{q} \cdot \mathbf{b}}\left\{e^{i \chi_{\mathrm{AI}}^{(0)}}-1\right\}, \\
\chi_{\mathrm{AI}}^{(0)}(\mathbf{b})=-\frac{m}{K} \int_{-\infty}^{+\infty} d z V\left(\mathbf{b}+\hat{\mathbf{K}}_{z} z\right),
\end{gathered}
$$

because the functional integrals are trivially one by normalization [34]. This is a variant of the eikonal approximation due to Abarbanel and Itzykson [35] where $K=k \cos (\theta / 2)$ appears everywhere instead of the asymptotic momentum $k$. For a spherically symmetric potential $V(r)$ we have the standard result

$$
\chi_{\mathrm{AI}}^{(0)}(b)=-\frac{2 m}{K} \int_{0}^{\infty} d z V\left(\sqrt{b^{2}+z^{2}}\right) .
$$

It is easy to calculate the next-to-leading order correction by expanding the phase up to linear order in $\mathbf{v}(t)$ and $\mathbf{w}(t)$, and performing the shifted Gaussian integral by means of Eq. (59). The result is

$$
T_{i \rightarrow f} \simeq T_{\mathrm{AI}}^{(1)}=i \frac{K}{m} \int d^{2} b e^{-i \mathbf{q} \cdot \mathbf{b}}\left\{\exp \left[i \chi_{\mathrm{AI}}^{(0)}+i \chi_{\mathrm{AI}}^{(1)}\right]-1\right\}
$$

with an additional phase function

$$
\begin{aligned}
\chi_{\mathrm{AI}}^{(1)}(\mathbf{b})= & -\frac{1}{8 m} \lim _{T \rightarrow \infty} \int_{-T}^{+T} d s \int d t_{1} d t_{2} \nabla V_{1} \cdot \nabla V_{2} \\
& \times\left[\operatorname{sgn}\left(t_{1}-s\right) \operatorname{sgn}\left(t_{2}-s\right)-\operatorname{sgn}^{2}(-s)\right] \\
= & \frac{1}{4 m} \int_{-\infty}^{+\infty} d t_{1} d t_{2} \nabla V_{1} \cdot \nabla V_{2}\left|t_{1}-t_{2}\right|,
\end{aligned}
$$

where $\nabla V_{i}$ is an abbreviation for $\nabla V\left(\mathbf{b}+\hat{\mathbf{K}} z_{i}\right)$. Again the con- tribution from the antivelocity $\mathbf{w}$ naturally cancels explicit $T$ terms when the integration over $s$ is performed with the help of Eq. (39). In Appendix B it is shown that for a spherically symmetric potential the expression simplifies to

$$
\chi_{\mathrm{AI}}^{(1)}(b)=-\frac{1}{K}\left(\frac{m}{K}\right)^{2}\left[1+b \frac{\partial}{\partial b}\right] \int_{0}^{\infty} d z V^{2}(r), \quad r \equiv \sqrt{b^{2}+z^{2}} .
$$

This is identical with the phase $\tau_{1}(b)$ in the systematic eikonal expansion of Wallace [14] apart from the appearance of $K=k \cos (\theta / 2)$ instead of $k$ which is unimportant in this order and for forward direction. Note that this additional phase already appears in exponentiated form as conjectured by Wallace.

One may wonder whether Eq. (74) is the correct result up to order $K^{-1}$ because the next order term is also of that order. For insight into this question it is instructive to consider the example of an one-dimensional (ordinary) integral

$$
\mathcal{T}(a, \epsilon):=\frac{\int d x \exp \left(i x^{2}\right) \exp [-i V(a+\sqrt{\epsilon} x)]}{\int d x \exp \left(i x^{2}\right)} \equiv\left\langle e^{i V(a+\sqrt{\epsilon} x)}\right\rangle .
$$

After expanding the function $V$ in the exponent for small $\epsilon$ as $V(a+\sqrt{\epsilon} x)=V(a)+\sqrt{\epsilon} V^{\prime}(a) x+\cdots$, keeping terms up to order $x^{2}$ in the exponent, expanding higher-order terms, integrating term by term and re-exponentiating one obtains

$$
\begin{aligned}
\mathcal{T}(a, \epsilon)= & \mathcal{N} \int d x \exp \left\{-i V(a)+i x^{2}\left[1-\epsilon V^{\prime \prime}(a) / 2\right]\right. \\
& \left.-i x \sqrt{\epsilon} V^{\prime}(a)\right\}\left[1+O\left(\epsilon^{3 / 2} x^{3}\right)\right] \\
= & \exp \left[-i V(a)-\frac{i \epsilon}{4\left(1-\epsilon V^{\prime \prime}(a) / 2\right)} V^{\prime 2}(a)\right. \\
& \left.-\frac{1}{2} \ln \left(1-\epsilon V^{\prime \prime}(a) / 2\right)\right]\left\{1+O\left(\epsilon^{2}\right)\right\} \\
= & \exp \left[-i V(a)-i \frac{\epsilon}{4} V^{\prime 2}(a)+\frac{\epsilon}{4} V^{\prime \prime}(a)+O\left(\epsilon^{2}\right)\right] .
\end{aligned}
$$

Since the correction phase $\chi_{\mathrm{AI}}^{(1)}$ was obtained by truncating the Taylor expansion of the potential at first order it just corresponds to the second term and we seem to have missed another, purely imaginary phase linear in the potential which is also first order in $\epsilon$ or $1 / K$. However, closer examination shows that this is not the case. It is, of course, possible to prove that assertion directly by evaluating the required functional integrals. These are more general Gaussian integrals of the type 


$$
\begin{aligned}
\mathcal{N} \int & \mathcal{D} v \exp \left\{i \int_{-T}^{+T} d t\left[\frac{m}{2} v^{2}(t)+\sqrt{\epsilon} g(t) v(t)\right]\right. \\
& \left.+\epsilon \int_{-T}^{+T} d t d t^{\prime} v(t) h\left(t, t^{\prime}\right) v\left(t^{\prime}\right)\right\} \\
& =\exp \left[-i \frac{\epsilon}{2 m} \int_{-T}^{+T} d t g^{2}(t)-\frac{\epsilon}{m} \int_{-T}^{+T} d t h(t, t)+O\left(\epsilon^{2}\right)\right]
\end{aligned}
$$

and multidimensional extensions thereof. However, there is an easier approach using the cumulant expansion (see, for example, Ref. [36]) which in the one-dimensional example of Eq. (75) reads

$$
\begin{gathered}
\mathcal{T}(a, \epsilon)=\exp \left[i c_{1}(a, \epsilon)+\frac{i^{2}}{2 !} c_{2}(a, \epsilon)+\cdots\right], \\
c_{1}(a, \epsilon)=\epsilon\langle V(a+\sqrt{\epsilon} x)\rangle, \\
c_{2}(a, \epsilon)=\epsilon^{2}\left\langle[V(a+\sqrt{\epsilon} x)-\langle V(a+\sqrt{\epsilon} x)\rangle]^{2}\right\rangle, \ldots .
\end{gathered}
$$

Of course, by expanding the cumulants in powers of $\epsilon$ one obtains the same result (76) as before. Application to the eikonal expansion is straightforward: it is easy to calculate the cumulants in closed form and since $\chi_{\mathrm{AI}}^{(1)}$ is quadratic in the potential we only have to expand the first cumulant in inverse powers of $K$ in order to obtain all terms which are linear in the potential. This is very similar to working out the first Born approximation and we obtain

$$
\begin{aligned}
-\int_{-\infty}^{+\infty} & d s\left\langle V\left[\xi_{\mathbf{K}}(s)\right]\right\rangle=-\int \frac{d^{3} p}{(2 \pi)^{3}} \tilde{V}(\mathbf{p}) \\
& \times \int_{-\infty}^{+\infty} d s e^{i \mathbf{p} \cdot[\mathbf{b}+(\mathbf{K} / m) s]}\left\langle e^{i \mathbf{p} \cdot\left[\mathbf{x}_{v}(s)-\mathbf{x}_{w}(0)\right]}\right\rangle \\
= & -\int \frac{d^{3} p}{(2 \pi)^{3}} \widetilde{V}(\mathbf{p}) \int_{-\infty}^{+\infty} d s \\
& \times \exp \left[-i \mathbf{p} \cdot\left(\mathbf{b}+\frac{\mathbf{K}}{m} s\right)\right] \\
= & -\frac{m}{K} \int_{-\infty}^{+\infty} d z V(\mathbf{b}+\hat{K} z) \equiv \chi_{\mathrm{AI}}^{(0)}(b),
\end{aligned}
$$

where the (functional) average over $\mathbf{v}, \mathbf{w}$ with the weight $\exp \left[\operatorname{im} \int d t\left(\mathbf{v}^{2}-\mathbf{w}^{2}\right) / 2\right]$ gives one according to Eq. (60). Hence there are no higher-order terms linear in the potential beyond the leading eikonal phase and Eqs. (72) and (73) are correct up to and including order $1 / K$.

\section{B. Ray expansion}

The path integral representation (44) gives rise to a different high-energy expansion because we expand around the momentum $\mathbf{p}_{\text {ray }}(t)$ which takes into account the different asymptotic directions before and after the scattering. While this complicates the analysis and leads to an additional mo- mentum transfer dependence some advantages at larger scattering angles may be expected: Applying a similar scaling argument as in Eqs. (66) and (67) one sees that now a systematic expansion in inverse powers of $k$ is obtained which we will call the "ray" expansion. A disadvantage is the expansion around a discontinuous reference path which abruptly changes direction at $t=0$. This may deteriorate the convergence properties of the expansion but may be remedied by another choice of the function $f(t)$ in Eq. (18) subject to the constraint (17).

The lowest order term is obtained by setting $\mathbf{v}=0$ in the argument of $V$ and immediately gives a new high-energy approximation

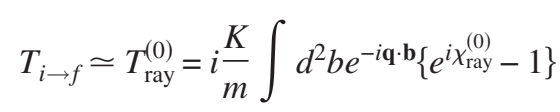

with a phase [37]

$$
\begin{gathered}
\chi_{\text {ray }}^{(0)}(\mathbf{b}, \mathbf{q})=-\frac{m}{k} \int_{-\infty}^{+\infty} d z V(\boldsymbol{\rho}(z)), \\
\boldsymbol{\rho}(z)=\mathbf{b}+\frac{\mathbf{p}(t=m z / k)}{k} z=\mathbf{b}+\frac{\mathbf{q}}{2 k}|z|+\frac{\mathbf{K}}{k} z \\
\chi_{\text {ray }}^{(0)}(\mathbf{b}, \mathbf{q}) \equiv-\frac{m}{k} \int_{0}^{\infty} d z\left[V\left(\mathbf{b}-\hat{\mathbf{k}}_{i} z\right)+V\left(\mathbf{b}+\hat{\mathbf{k}}_{f} z\right)\right] .
\end{gathered}
$$

This has some similarity with the eikonal phase derived by Lévy and Sucher [17] although these "symmetric" eikonal expansions [38] are quite different from our approach.

For a spherically symmetric potential we have for the leading order ray phase function

$$
\chi_{\text {ray }}^{(0)}(b, \beta)=-\frac{2 m}{k} \int_{0}^{\infty} d z V(\rho(z)),
$$

where

$$
\rho(z)=\sqrt{b^{2}+z^{2}+\mathbf{b} \cdot \mathbf{q} z / k}=\sqrt{b^{2}+z^{2}+2 b z \beta} .
$$

Here we have defined

$$
\beta=\frac{\hat{\mathbf{b}} \cdot \mathbf{q}}{2 k}=\sin \left(\frac{\theta}{2}\right) \cos \varphi, \quad|\beta| \leqslant 1,
$$

where $\varphi$ is the angle between the impact parameter and the momentum transfer. In forward direction (where all different eikonal approximations should be equivalent) this is seen to reduce to the usual eikonal phase plus a correction:

$$
\chi_{\mathrm{ray}}^{(0)}(b, \beta) \stackrel{\theta \rightarrow 0}{\rightarrow}-\frac{2 m}{k} \int_{0}^{\infty} d z V(r)-\frac{2 m}{k} \frac{\mathbf{b} \cdot \mathbf{q}}{2 k} \int_{0}^{\infty} d z \frac{z}{r} V^{\prime}(r)+\cdots .
$$

With $\partial V(r) / \partial z=z V^{\prime}(r) / r$ the correction term can be easily integrated and gives 


$$
\chi_{\text {ray }}^{(0)}(\mathbf{b}, \beta) \stackrel{\theta \rightarrow 0}{\rightarrow}-\frac{2 m}{k} \int_{0}^{\infty} d z V(r)+\frac{m}{k^{2}} \frac{\mathbf{b} \cdot \mathbf{q}}{2 k} V(b)+\cdots
$$

Combining the result with the $\exp (-i \mathbf{q} \cdot \mathbf{b})$ factor in the impact parameter integral it is thus seen that the main effect of the ray approximation is the replacement of the momentum transfer by an effective momentum transfer

$$
q_{\mathrm{eff}}(b)=q\left(1-\frac{m}{k^{2}} V(b)\right),
$$

which takes into account the energy gained (or lost) by moving in the attractive (or repulsive) potential at closest approach:

$$
\frac{k^{2}}{2 m}=\frac{k_{\mathrm{eff}}^{2}(b)}{2 m}+V(b, z=0), \quad q_{\mathrm{eff}}=2 k_{\mathrm{eff}} \sin \left(\frac{\theta}{2}\right) .
$$

This approximation (with an average, constant value of the potential) is standard practice in electron scattering from nuclei where higher order effects are roughly included by evaluating the Born approximation form factor as function of an effective momentum transfer [39]. However, when doing that it is also well known [40] that a flux factor $\left(k_{\mathrm{eff}} / k\right)^{2}$ is needed for the scattering amplitude.

This flux factor is provided by a purely imaginary phase $\omega_{\text {ray }}^{(1)}$ which appears in next-to-leading order and corresponds to the second term in the example (76). In contrast to the eikonal expansion in the previous subsection this correction does not vanish anymore. Let us evaluate it by calculating the first cumulant:

$$
\begin{aligned}
\left\langle\chi_{\text {ray }}\right\rangle= & -\int \frac{d^{3} p}{(2 \pi)^{3}} \tilde{V}(\mathbf{p}) \int_{-\infty}^{+\infty} d s \exp [-i \mathbf{p} \cdot \boldsymbol{\rho}(s)] \\
& \times\left\langle\exp \left\{-i \mathbf{p} \cdot\left[\mathbf{x}_{v}(s)-\mathbf{x}_{v \perp}(0)\right]+i \mathbf{p} \cdot \hat{\mathbf{K}} x_{w}(0)\right\}\right\rangle .
\end{aligned}
$$

The average in the last line has already been evaluated in Eq. (64) so that

$$
\begin{aligned}
\left\langle\chi_{\text {ray }}\right\rangle= & -\frac{m}{k} \int_{-\infty}^{+\infty} d z \int \frac{d^{3} p}{(2 \pi)^{3}} \tilde{V}(\mathbf{p}) \\
& \times \exp [-i \mathbf{p} \cdot \boldsymbol{\rho}(z)] \exp \left[-i \frac{\mathbf{p}_{\perp}^{2}}{2 k}|z|\right] \\
= & \chi_{\text {ray }}^{(0)}+i \omega_{\text {ray }}^{(1)}+O\left(k^{-2}\right) .
\end{aligned}
$$

To order $k^{-1}$ there is now a purely imaginary phase with magnitude

$$
\begin{aligned}
\omega_{\text {ray }}^{(1)}(\mathbf{b}, \mathbf{q}) & =\frac{m}{2 k^{2}} \int_{-\infty}^{+\infty} d z|z| \int \frac{d^{3} p}{(2 \pi)^{3}} \tilde{V}(\mathbf{p}) \mathbf{p}_{\perp}^{2} \exp [-i \mathbf{p} \cdot \rho(z)] \\
& =-\frac{m}{2 k^{2}} \Delta_{b} \int_{-\infty}^{+\infty} d z|z| V(\boldsymbol{\rho}(z))
\end{aligned}
$$

where

$$
\Delta_{b}=\frac{\partial^{2}}{\partial b^{2}}+\frac{1}{b} \frac{\partial}{\partial b}+\frac{1}{b^{2}} \frac{\partial^{2}}{\partial \varphi^{2}}=\frac{1}{b} \frac{\partial}{\partial b} b \frac{\partial}{\partial b}+\frac{1}{b^{2}} \frac{\partial^{2}}{\partial \varphi^{2}} .
$$

For a spherically symmetric potential this simplifies to

$$
\omega_{\text {ray }}^{(1)}(b, \beta, \theta)=-\frac{1}{k} \frac{m}{k} \Delta_{b} \int_{0}^{\infty} d z z V(\boldsymbol{\rho}(z)),
$$

Note that $\omega_{\text {ray }}^{(1)}$ now depends on three variables (apart from the overall powers of $1 / k): b, \mathbf{b} \cdot \mathbf{q}, q$ or $b, \beta, \theta$. This is because the Laplacian $\Delta_{b}$ contains explicit derivatives with respect to $\varphi$.

What is the effect of the real factor

$$
e^{-\omega_{\text {ray }}^{(1)}} \simeq 1-\omega_{\text {ray }}^{(1)}
$$

on the scattering amplitude? If the improvement from the leading ray approximation is incorporated into an effective momentum transfer (as discussed above) we may consider $\exp \left(-\omega_{\text {ray }}^{(1)}\right)$ simply as an amplitude correction as discussed above. However, an alternative interpretation arises if the correction (88) is included in a scaled impact parameter

$$
b^{\prime}=b\left(1-\frac{m}{k^{2}} V(b)\right) \Rightarrow b=b^{\prime}\left(1+\frac{m}{k^{2}} V\left(b^{\prime}\right)\right)+O\left(\frac{1}{k^{4}}\right)
$$

This implies the following change in the integration measure

$$
b d b=b^{\prime} d b^{\prime}\left[1+\frac{m}{k^{2}}\left(V\left(b^{\prime}\right)+\frac{d}{d b^{\prime}}\left[b^{\prime} V\left(b^{\prime}\right)\right]\right)\right] .
$$

However, in forward direction $\rho^{2}(z) \rightarrow r^{2}=b^{2}+z^{2}$ and therefore

$$
\begin{aligned}
\left.\omega_{\text {ray }}^{(1)}\right|_{\theta=0} & =-\frac{m}{k^{2}} \frac{1}{b} \frac{\partial}{\partial b} b \int_{0}^{\infty} d z z \frac{\partial V}{\partial b} \\
& =-\frac{m}{k^{2}} \frac{1}{b} \frac{\partial}{\partial b} b \int_{0}^{\infty} d z b \frac{\partial V}{\partial z}=\frac{m}{k^{2}}\left[2 V(b)+b V^{\prime}(b)\right]
\end{aligned}
$$

so that $\exp \left(-\omega_{\text {ray }}^{(1)}\right)$ exactly cancels (at least in the forward direction) the Jacobian arising from the scaling transformation. The leading order ray phase with the scaled impact parameter as argument is

$$
\begin{aligned}
\chi_{\text {ray }}^{(0)}\left(b^{\prime}, \beta \rightarrow 0\right) \simeq & -\frac{2 m}{k} \int_{0}^{\infty} d z V\left(r^{\prime}\right) \\
& -\frac{2 m^{2}}{k^{3}} b^{\prime 2} V\left(b^{\prime}\right) \int_{0}^{\infty} d z \frac{V^{\prime}\left(r^{\prime}\right)}{r^{\prime}} \\
= & -\frac{2 m}{k} \int_{0}^{\infty} d z V\left(r^{\prime}\right) \\
& -\frac{2 m^{2}}{k^{3}} V\left(b^{\prime}\right) b^{\prime} \frac{\partial}{\partial b^{\prime}} \int_{0}^{\infty} d z V\left(r^{\prime}\right),
\end{aligned}
$$




$$
r^{\prime}=\sqrt{b^{\prime 2}+z^{2}}
$$

which has a correction term similar to Eq. (74) in the eikonal expansion. Thus the leading order ray expansion already contains approximately higher order eikonal terms.

Of course, there is also a real first-order phase $\chi_{\text {ray }}^{(1)}$ which is obtained by expanding $\chi_{\text {ray }}$ up to first order in $\mathbf{v}, w$ :

$$
\chi_{\text {ray }}(\mathbf{b}, \mathbf{v}, w)=\chi_{\text {ray }}^{(0)}+\int_{-T}^{+T} d t\left[\mathbf{g}_{v}(t) \cdot \mathbf{v}(t)-g_{w}(t) w(t)\right]+\cdots,
$$

where now

$$
\left[\mathbf{g}_{v}(t)\right]_{k}=-\frac{1}{2} \int_{-T}^{+T} d t_{1} \partial_{k} V\left(\rho_{1}\right)\left[\operatorname{sgn}\left(t_{1}-t\right)-\left(1-\delta_{k 3}\right) \operatorname{sgn}(-t)\right],
$$

$$
g_{w}(t)=\frac{1}{2} \int_{-T}^{+T} d t_{1} \partial_{k} V\left(\rho_{1}\right) \operatorname{sgn}(-t)
$$

Here $k=1,2,3$ are the Cartesian coordinates of the vector $\mathbf{g}_{v}$ and the argument of the potential is always $\boldsymbol{\rho}_{1}=\mathbf{b}+\mathbf{x}_{\text {ref }}\left(t_{1}\right)$ $=\mathbf{b}+\mathbf{K} t_{1} / m+\mathbf{q}\left|t_{1}\right| /(2 m)$. Applying the Gaussian integration formula (59) we obtain the real correction phase of order one

$$
\chi_{\text {ray }}^{(1)}(\mathbf{b}, \mathbf{q})=-\frac{1}{2 m} \int_{-T}^{+T} d t\left[\mathbf{g}_{v}^{2}(t)-g_{w}^{2}(t)\right]
$$

and performing the $t$ integration with the help of Eq. (39) we find-as expected-that all $T$ dependence cancels. Thus

$$
\begin{aligned}
\chi_{\text {ray }}^{(1)}(\mathbf{b}, \mathbf{q})= & \frac{1}{4 m} \int_{-\infty}^{+\infty} d t_{1} d t_{2}\left\{\nabla V\left(t_{1}\right) \cdot \nabla V\left(t_{2}\right)\left|t_{1}-t_{2}\right|\right. \\
& \left.-\nabla_{\mathbf{b}} V\left(t_{1}\right) \cdot \nabla_{\mathbf{b}} V\left(t_{2}\right)\left(\left|t_{1}\right|+\left|t_{2}\right|\right)\right\} .
\end{aligned}
$$

For a spherically symmetric potential some algebra which is outlined in Appendix B leads to

$$
\begin{aligned}
\chi_{\text {ray }}^{(1)}(b, \beta)= & -\frac{1}{k} \frac{m^{2}}{k^{2}}\left\{\left(1+b \frac{\partial}{\partial b}\right) \int_{0}^{\infty} d z V^{2}(\rho)\right. \\
& -\frac{b}{1-\beta^{2}}\left[2 V(b) \frac{\partial}{\partial b} \int_{0}^{\infty} d z V(\rho)+\beta V^{2}(b)\right. \\
& \left.\left.+\beta\left(\frac{\partial}{\partial b} \int_{0}^{\infty} d z V(\rho)\right)^{2}\right]\right\},
\end{aligned}
$$

where $\rho$ and $\beta$ are defined in Eq. (85) and (86), respectively. The first term in Eq. (106) is identical with Wallace's eikonal phase $\tau_{1}$ for forward scattering when $\rho \rightarrow r$. It may be surprising that Eq. (106) contains an additional term which does not vanish in forward direction, i.e., for $\beta=0$. But this is just the term which exactly cancels the last term in the approximation of Eq. (100) so that the correct first-order eikonal expression for forward scattering is obtained. Note that there is no singularity in Eq. (106) at $\beta= \pm 1$ as can be also seen in Appendix C. We therefore have in first-order ray expansion

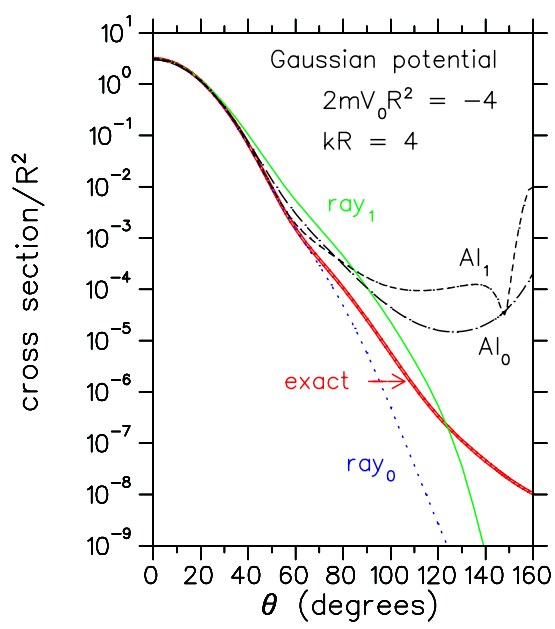

FIG. 2. (Color online) Differential cross section from a Gaussian potential with strength $2 m V_{0} R^{2}=-4$ at $k R=4$ as function of the scattering angle $\theta$. Shown are the exact result from a partial wave calculation and from the zeroth and first order of the high-energy expansions derived in Sec. VI. In these the particle travels along the mean momentum [an eikonal approximation due to Arbabanel and Itzykson (AI)] or along a ray made up of the initial and the final momentum.

$$
T_{i \rightarrow f} \simeq T_{\text {ray }}^{(1)}=\frac{K}{m} \int d^{2} b e^{-i \mathbf{q} \cdot \mathbf{b}}\left\{\exp \left[i \chi_{\text {ray }}^{(0)}+i \chi_{\text {ray }}^{(1)}-\omega_{\text {ray }}^{(1)}\right]-1\right\}
$$

\section{NUMERICAL RESULTS}

Let us test the high-energy expansions for the case of scattering from a Gaussian potential

$$
V(r)=V_{0} e^{-r^{2} / R^{2}}
$$

with the parameter values $2 m V_{0} R^{2}=-4, k R=4$, i.e., $E=-4 V_{0}$, corresponding to the case where $\alpha$ particles scatter elastically from $\alpha$ particles at $166 \mathrm{MeV}$ center-of-mass energy $(R=1 \mathrm{fm})$. The parameters are precisely those where convergence of the standard eikonal expansion was found to be unsatisfactory [14]. For completeness the analytical expressions for the various phases of a Gaussian potential are listed in Appendix C. We have evaluated $T_{\mathrm{AI}}$ and $T_{\text {ray }}$ by numerical integration using Gauss-Legendre rules with 72 points and a sufficient number of subdivisions of the integration interval which was mapped to a finite range by $y_{i}$ $=R \tan \psi_{i}$ where $y \equiv b, z$ for the $\mathrm{AI}$ expansion and $y$ $\equiv b_{x}, b_{y}, z$ for the ray expansion. Figure 2 shows the differential cross section obtained from these high-energy approximations compared to an exact partial wave calculation. The (AI) eikonal expansion shows the well-known failure at larger scattering angles and the corrections only slightly increase the point of deviation [41]. Since the cross section is sharply peaked in forward direction the total cross section is always well reproduced despite the deviations at higher scattering angles and not suited as a measure of (dis)agreement.

The ray expansion does better at higher scattering angles at the price of being more complicated and less precise at 


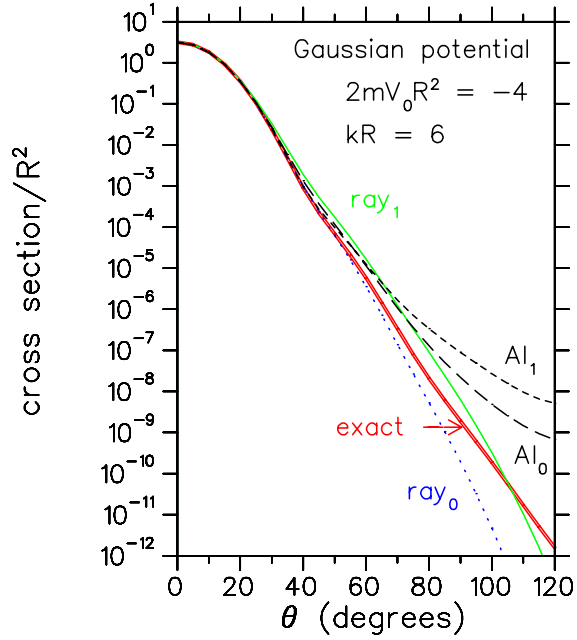

FIG. 3. (Color online) Same as in Fig. 2 but for $k R=6$.

small scattering angles. As scattering from a Gaussian potential at larger scattering angles is known to be dominated by many small scatterings these deficiencies may be attributed to the sudden change at $t=0$ which imparts a large momentum transfer to the scattered particle. In addition, as was mentioned before a derivative expansion about this discontinuous path will probably run into problems. It may be expected that a description based on a smooth path will do better but this will not be pursued in the present work.

Figure 3 shows how the different expansions describe the cross section at higher energy $(k R=6)$. Again the ray expansion is closer to the exact result at higher scattering angles.

\section{SUMMARY AND OUTLOOK}

Time-dependent methods for scattering have been investigated by several authors [42] in ordinary quantum mechanics. Using path integrals over velocities I have derived two new representations for the nonrelativistic $T$ matrix which in a very natural way describe the propagation of high-energy particles in a local potential. Although the time evolution of the scattering process is also central in the present approach it leads to formulations which are quite different from the previous ones. This is because two important requirements must be fulfilled for obtaining a path integral formulation of the $T$ matrix from the matrix elements of the time-evolution operator $\hat{U}(T,-T)$ for infinite scattering times $T$. First one has to make sure that phases are eliminated which diverge for $T \rightarrow \infty$ and, second, a suitable constraint has to be found which leads to energy conservation in the $S$ matrix. In the present paper the first requirement is met by introducing phantom degrees of freedom ("antivelocity") which cancel these divergences in a way reminiscent of the Lee-Wick proposal for quantum field theory. Energy conservation is achieved by using the classic Faddeev-Popov procedure such that the component of the position vector parallel to the mean momentum $\mathbf{K}=\left(\mathbf{k}_{i}+\mathbf{k}_{f}\right) / 2$ is fixed. This involved some delicate (and at present not very well-controlled) limit procedures but we have checked that the resulting path-integral formulations of the $T$ matrix produce the correct Born series in all orders.

One of the advantages of these new path-integral formulations is that they can give rise to new approximation schemes or expansions. As they are close to a geometrical picture of scattering where the path integral describes the quantum fluctuations around some reference path it is not surprising that the eikonal approximation (in the variant of Abarbanel and Itzykson [35] where the particle travels along a straight-line path with velocity $\mathbf{K} / \mathrm{m}$ ) immediately follows and that corrections to it can be calculated systematically. A suitable scaling of variables in the path integral shows that these corrections involve inverse powers of $K=k \cos (\theta / 2)$ and therefore inevitably grow at larger scattering angles $\theta$. However, one is also naturally led to a new variant (ray approximation) which displays the different asymptotic directions along which the particle propagates at high energy and which should work at high energy irrespective of the scattering angle. Indeed, for high-energy scattering from a Gaussian potential some improvement over the AbarbanelItzykson eikonal expansion was achieved.

There seems to be considerable room (and need) for improvement: a better Faddeev-Popov constraint should eliminate the rather asymmetric treatment of longitudinal and perpendicular variables. It is unclear how Wallace's eikonal expansion [14] (where the particle is traveling along the mean momentum but with velocity $\mathrm{k} / \mathrm{m}$ ) could emerge naturally from a path-integral representation. This formulation gives an impact-parameter representation of the $T$ matrix and produces the exact Coulomb amplitude in lowest order [43] — which is not the case for the present formulation. A better control of the delicate limit $T \rightarrow \infty$ needed for obtaining the $S$ matrix is certainly desired and finally one may ask whether a formulation without phantom degrees of freedom is possible.

However, despite these shortcomings and the long list of desiderata our formulation seems to have some merits: at least it enlarges the "tool-box" of scattering theory and offers new possibilities. Among these one may expect new approximation schemes and, hopefully the prospect of evaluating the real-time path integral numerically, i.e., achieving a stochastic evaluation of the scattering process. Obviously this would be of great importance in the many-body case where one may assume the interaction potential as

$$
V(\mathbf{r})=\sum_{k=1}^{N} V\left(\mathbf{r}-\mathbf{r}_{k}\right),
$$

with $\mathbf{r}_{k}$ denoting the position of the $k$ th scatterer. It is amusing that the path integral representations discussed in this paper lead to a multiple scattering expansion with exactly $N$ terms when

$$
\begin{aligned}
\exp \left(i \sum_{k=1}^{N} \chi_{k}\right) & =\prod_{k=1}^{N}\left[1+\left(e^{i \chi_{k}}-1\right)\right] \\
& =1+\sum_{j=1}^{N} \sum_{k_{1}<k_{2}<\ldots k_{j}} \prod_{l=1}^{j}\left[\exp \left(i \chi_{k_{l}}\right)-1\right]
\end{aligned}
$$

is used. This is in contrast to Watson's multiple scattering 
expansion (see, e.g., Ref. [44]) which contains infinite many terms and much closer to Glauber's theory where the incident particle cannot scatter back due to its straight-line propagation. However, because of the subsequent path integration over all velocities the present expansion (if taken to full order) is not an approximation but allows for repeated scattering from the same scattering center.

Extensions to relativistic scattering [45] also seem possible. Further investigations of this formulation as well as numerical studies of the real-time path integral will be reported elsewhere [46].

\section{ACKNOWLEDGMENTS}

I would like to thank Dina Alexandrou for many discussions and helpful remarks in the early stages of this work which remained dormant for a long time. I am also grateful to H. Kleinert for his interest and for including some results in the latest version of his textbook. Finally I am indebted to a referee for well-founded suggestions and a meticulous checking of text and formulas.

\section{APPENDIX A: COMPLETE BORN SERIES FROM THE PATH-INTEGRAL REPRESENTATIONS}

Here we show that the various path-integral representations for the $T$ matrix exactly reproduce the conventional Born series to all orders if the exponent is expanded in powers of the potential

$$
\begin{aligned}
\exp \left[-i \int_{-T}^{+T} d t V(\boldsymbol{\xi}(t))\right]-1 \\
\quad=\sum_{n=1}^{\infty} \frac{(-i)^{n}}{n !} \int_{-T}^{+T} d t_{1} \cdots d t_{n} V\left(\boldsymbol{\xi}\left(t_{1}\right)\right) \cdots V\left(\boldsymbol{\xi}\left(t_{n}\right)\right)
\end{aligned}
$$

and the functional integrations are done term by term. This can be done by Fourier transforming the potential

$$
V\left(\boldsymbol{\xi}\left(t_{i}\right)\right)=\int \frac{d^{3} p_{i}}{(2 \pi)^{3}} \tilde{V}\left(\mathbf{p}_{i}\right) e^{i \mathbf{p}_{i} \cdot \boldsymbol{\xi}\left(t_{i}\right)} .
$$

We do that first for the version with a three-dimensional antivelocity as given in Eqs. (27) and (29), where the reference path is along the average momentum

$$
\mathbf{x}_{\mathrm{ref}}^{\mathrm{eik}}\left(t_{i}\right)=\frac{\mathbf{K}}{m} t_{i}, \boldsymbol{\xi}\left(t_{i}\right) \equiv \boldsymbol{\xi}_{\mathbf{K}}\left(t_{i}\right)=\mathbf{b}+\mathbf{x}_{\mathrm{ref}}^{\mathrm{eik}}\left(t_{i}\right)+\mathbf{x}_{v}\left(t_{i}\right)-\mathbf{x}_{w}(0) .
$$

We then obtain

$$
T_{i \rightarrow f}=: \sum_{n=1}^{\infty} T_{n}
$$

with

$$
\begin{aligned}
T_{n}^{(3-d)}= & i \frac{K}{m} \frac{(-i)^{n}}{n !} \int d^{2} b e^{-i \mathbf{q} \cdot \mathbf{b}} \prod_{i=1}^{n}\left(\int_{-T}^{+T} d t_{i} \int \frac{d^{3} p_{i}}{(2 \pi)^{3}} \tilde{V}\left(\mathbf{p}_{i}\right)\right) \\
& \times \exp \left[i \sum_{i=1}^{n} \mathbf{p}_{i} \cdot\left(\mathbf{b}+\mathbf{x}_{\mathrm{ref}}\left(t_{i}\right)\right)\right] G_{n}^{(3-d)}
\end{aligned}
$$

For $d=3$ we have to evaluate

$$
\begin{aligned}
G_{n}^{(3-3)} & =|\mathcal{N}|^{6} \int \mathcal{D}^{3} v \mathcal{D}^{3} w \exp \left[i \int_{-T}^{+T} d t \frac{m}{2}\left(\mathbf{v}^{2}-\mathbf{w}^{2}\right)\right] \\
& \times \exp \left[i \sum_{i=1}^{n} \mathbf{p}_{i} \cdot\left[\mathbf{x}_{v}\left(t_{i}\right)-\mathbf{x}_{w}(0)\right]\right]
\end{aligned}
$$

Since

$$
\mathbf{x}_{v}\left(t_{i}\right)-\mathbf{x}_{w}(0)=\frac{1}{2} \int_{-T}^{+T} d t\left[\operatorname{sgn}\left(t_{i}-t\right) \mathbf{v}(t)-\operatorname{sgn}(-t) \mathbf{w}(t)\right]
$$

the path integrals to be evaluated are just Gaussian integrals of the same form as in Eq. (59) giving the result

$$
\begin{aligned}
G_{n}^{(3-3)}= & \exp \left\{-\frac{i}{8 m} \sum_{i, j=1}^{n} \mathbf{p}_{i} \cdot \mathbf{p}_{j} \int_{-T}^{+T} d t\left[\operatorname{sgn}\left(t_{i}-t\right) \operatorname{sgn}\left(t_{j}-t\right)\right.\right. \\
& \left.\left.-\operatorname{sgn}^{2}(-t)\right]\right\}
\end{aligned}
$$

Note that the first term in the square bracket comes from the functional integration over $\mathbf{v}$ and the second one from the functional integration over the antivelocity. As usual we have regulated the time integration by a finite time $T$ which we finally will send to infinity. Using Eq. (39) any divergence in this limit is cancelled by the contribution from the antivelocity

$$
G_{n}^{(3-3)}=\exp \left\{-\frac{i}{4 m} \sum_{i, j=1}^{n} \mathbf{p}_{i} \cdot \mathbf{p}_{j}\left(T-\left|t_{i}-t_{j}\right|-T\right)\right\},
$$

as was expected.

Next we consider the ray representation (44) with an onedimensional antivelocity which is a little bit more involved: first, the reference path is

$$
\mathbf{x}_{\mathrm{ref}}^{\mathrm{ray}}(t)=\frac{\mathbf{p}_{\mathrm{ray}}}{m} t=\frac{\mathbf{K}}{m} t+\frac{\mathbf{q}}{2 m}|t| .
$$

Second, the path integrals to be performed are again of the form (59) but with

$$
\mathbf{g}_{v \perp}(t)=\frac{1}{2} \sum_{i=1}^{n} \mathbf{p}_{\perp i}\left[\operatorname{sgn}\left(t_{i}-t\right)-\operatorname{sgn}(-t)\right],
$$

$$
g_{v \|}(t)=\frac{1}{2} \sum_{i=1}^{n} p_{\| i} \operatorname{sgn}\left(t_{i}-t\right), \quad g_{w}(t)=\frac{1}{2} \sum_{i=1}^{n} p_{\| i} \operatorname{sgn}(-t)
$$

Therefore the Gaussian integration gives 


$$
G_{n}^{(3-1)}=\exp \left\{-\frac{i}{2 m} \int_{-T}^{+T} d t\left[\mathbf{g}_{v \perp}^{2}(t)+g_{v \|}^{2}(t)-g_{w}^{2}(t)\right]\right\}
$$

and after performing the $t$ integral by means of Eq. (39) and some algebra one obtains

$$
G_{n}^{(3-1)}=\exp \left\{\frac{i}{4 m} \sum_{i, j=1}^{n}\left[\mathbf{p}_{i} \cdot \mathbf{p}_{j}\left|t_{i}-t_{j}\right|-\mathbf{p}_{\perp i} \cdot \mathbf{p}_{\perp j}\left(\left|t_{i}\right|+\left|t_{j}\right|\right)\right]\right\} .
$$

Compared to Eq. (A9) there is an additional term which, however, is exactly cancelled by the additional term from

$$
\sum_{i=1}^{n} \mathbf{p}_{i} \cdot \frac{\mathbf{p}_{\mathrm{ray}}\left(t_{i}\right)}{m} t_{i}=\sum_{i=1}^{n} \mathbf{p}_{i} \cdot \frac{\mathbf{K}}{m} t_{i}+\sum_{i=1}^{n} \mathbf{p}_{i} \cdot \frac{\mathbf{q}}{2 m}\left|t_{i}\right|
$$

if one takes into account that the $\mathbf{b}$ integration enforces

$$
\sum_{i}^{n} \mathbf{p}_{\perp i}=\mathbf{q}
$$

Thus in both cases the $n$th order term in the Born series reads

$$
\begin{aligned}
T_{n}= & i \frac{K}{m} \frac{(-i)^{n}}{n !} \int d^{2} b e^{-i \mathbf{q} \cdot \mathbf{b}} \prod_{i=1}^{n}\left(\int_{-\infty}^{+\infty} d t_{i} \int \frac{d^{3} p_{i}}{(2 \pi)^{3}} \widetilde{V}\left(\mathbf{p}_{i}\right)\right) \\
& \times \exp \left[i \sum_{i=1}^{n} \mathbf{p}_{i} \cdot\left(\mathbf{b}+\frac{\mathbf{K}}{m} t_{i}\right)+\frac{i}{4 m} \sum_{i, j=1}^{n} \mathbf{p}_{i} \cdot \mathbf{p}_{j}\left|t_{i}-t_{j}\right|\right],
\end{aligned}
$$

where now the limit $T \rightarrow \infty$ has been taken. For further progress it is essential to recognize that the integrand is fully symmetric under exchange of $t_{i} \leftrightarrow t_{j}$ as can be verified by the corresponding exchange $\mathbf{p}_{i} \leftrightarrow \mathbf{p}_{j}$. If this is the case then

$$
\begin{aligned}
& \prod_{i=1}^{n}\left(\int_{-\infty}^{+\infty} d t_{i}\right) F_{\mathrm{symm}}\left(t_{1} \cdots t_{n}\right)=n ! \\
& \quad \times \int_{-\infty}^{+\infty} d t_{n} \int_{-\infty}^{t_{n}} d t_{n-1} \cdots \int_{-\infty}^{t_{2}} d t_{1} F_{\mathrm{symm}}\left(t_{1} \cdots t_{n}\right) .
\end{aligned}
$$

The factor in front cancels the factorial in the denominator of Eq. (A17). Furthermore, since the integration times are now ordered the last term in the exponential factor of Eq. (A17) becomes

$$
\begin{aligned}
\frac{i}{4 m} \sum_{i, j=1}^{n} \mathbf{p}_{i} \cdot \mathbf{p}_{j}\left|t_{i}-t_{j}\right| & =\frac{i}{2 m} \sum_{i<j}^{n} \mathbf{p}_{i} \cdot \mathbf{p}_{j}\left(t_{j}-t_{i}\right) \\
& =\frac{i}{2 m} \sum_{j=1}^{n} t_{j} \mathbf{p}_{j} \cdot \sum_{k=1}^{n} \operatorname{sgn}(j-k) \mathbf{p}_{k},
\end{aligned}
$$

where $\operatorname{sgn}(0)=0$ by convention. With the abbreviation

$$
u_{j}:=\frac{1}{2 m} \mathbf{p}_{j} \cdot \sum_{k=1}^{n} \operatorname{sgn}(j-k) \mathbf{p}_{k}+\mathbf{p}_{j} \cdot \frac{\mathbf{K}}{m}
$$

the time integrations can now be performed successively:

$$
\begin{gathered}
\int_{-\infty}^{t_{2}} d t_{1} e^{i t_{1}\left(u_{1}-i 0\right)}=\frac{-i}{u_{1}-i 0} e^{i t_{2}\left(u_{1}-i 0\right)}, \\
\int_{-\infty}^{t_{3}} d t_{2} e^{i t_{2}\left(u_{1}+u_{2}-i 0\right)} \\
=\frac{-i}{u_{1}+u_{2}-i 0} e^{i t_{3}\left(u_{1}+u_{2}-i 0\right)}, \ldots,
\end{gathered}
$$$$
\int_{-\infty}^{t_{n}} d t_{n-1} e^{i t_{n-1}\left(u_{1}+u_{2}+\cdots+u_{n-1}-i 0\right)}
$$$$
=\frac{-i}{u_{1}+u_{2}+\cdots+u_{n-1}-i 0}
$$$$
\times e^{i t_{n}\left(u_{1}+u_{2}+\cdots+u_{n-1}-i 0\right)}
$$

$$
\int_{-\infty}^{+\infty} d t_{n} e^{i t_{n} \Sigma_{j=1}^{n} u_{j}}=2 \pi \delta\left(\sum_{j=1}^{n} u_{j}\right) .
$$

Note that the prescription how to handle the singularities arises from the requirement that the time integrations should converge at the lower limit. Alternatively one could give the particle mass an infinitesimal imaginary part $m \rightarrow m+i 0$ already in the path integral so that $\exp \left(i \int_{-T}^{+T} d t m \mathbf{v}^{2} / 2\right)$ is damped.

Performing the $\mathbf{b}$ integration in Eq. (A17) gives another $\delta$ function so that

$$
\begin{aligned}
T_{n}= & i \frac{K}{m}(-i)^{n} \prod_{k=1}^{n}\left(\int \frac{d^{3} p_{k}}{(2 \pi)^{3}} \widetilde{V}\left(\mathbf{p}_{k}\right)\right) \prod_{k=1}^{n-1}\left(\frac{-i}{\sum_{j=1}^{k} u_{j}-i 0}\right) \\
& \times(2 \pi)^{2} \delta^{(2)}\left(\sum_{j=1}^{n} p_{j \perp}-\mathbf{q}\right) 2 \pi \delta\left(\sum_{j=1}^{n} u_{j}\right) .
\end{aligned}
$$

Recalling the definition of $u_{j}$ in Eq. (A20) we see that

$$
\sum_{j=1}^{n} u_{j}=\sum_{j=1}^{n} \mathbf{p}_{j} \cdot \frac{\mathbf{K}}{m}+\frac{1}{2 m} \sum_{j, k=1}^{n} \operatorname{sgn}(j-k) \mathbf{p}_{j} \cdot \mathbf{p}_{k}=\sum_{j=1}^{n} \mathbf{p}_{j} \cdot \frac{\mathbf{K}}{m}
$$

because the last term changes sign under the exchange $i \leftrightarrow j$. Thus 


$$
\begin{aligned}
T_{n}= & (2 \pi)^{3} \prod_{k=1}^{n}\left(\int \frac{d^{3} p_{k}}{(2 \pi)^{3}} \tilde{V}\left(\mathbf{p}_{k}\right)\right) \prod_{k=1}^{n-1}\left(\frac{1}{-\sum_{j=1}^{k} u_{j}+i 0}\right) \\
& \times \delta^{(2)}\left(\sum_{j=1}^{n} \mathbf{p}_{j \perp}-\mathbf{q}\right) \delta\left(\sum_{j=1}^{n} p_{j \|}\right) .
\end{aligned}
$$

For $n=1$ the last product is empty and the standard first-order Born approximation is obtained as already discussed in Sec. $\mathrm{V}$ B.

For $n>1$ the denominators in Eq. (A24) can be rewritten as

$$
-\sum_{j=1}^{k} u_{j}=\frac{1}{2 m} \sum_{j=1}^{k} \mathbf{p}_{j} \cdot\left(\sum_{i=k+1}^{n} \mathbf{p}_{i}-2 \mathbf{K}\right)
$$

and the $\delta$ functions allow us to replace

$$
\sum_{i=k+1}^{n} \mathbf{p}_{i \perp}=\mathbf{q}-\sum_{i=1}^{k} \mathbf{p}_{i \perp}, \quad \sum_{i=k+1}^{n} p_{i \|}=-\sum_{i=1}^{k} p_{i \|} .
$$

After some algebra one then obtains

$$
-\sum_{j=1}^{k} u_{j}=E-\frac{1}{2 m}\left[\left(\sum_{j=1}^{k} p_{i \|}+K\right)^{2}+\left(\sum_{i=1}^{k} \mathbf{p}_{i \perp}-\frac{\mathbf{q}}{2}\right)^{2}\right]
$$

with $E=k^{2} /(2 m)=\mathbf{k}_{i}^{2} /(2 m)=\mathbf{k}_{f}^{2} /(2 m)$. This suggests the transformation of integration variables to

$$
\mathbf{l}_{k}:=\left(\sum_{i=1}^{k} \mathbf{p}_{i \perp}-\frac{\mathbf{q}}{2}, \sum_{j=1}^{k} p_{i \|}+K\right),
$$

so that

$$
\mathbf{p}_{k}=\mathbf{l}_{k}-\mathbf{l}_{k-1}, \quad k=2 \cdots n .
$$

This also holds for $k=1$ if we define

$$
\mathbf{l}_{0}:=\left(-\frac{\mathbf{q}}{2}, K\right)=\mathbf{K}-\frac{\mathbf{q}}{2} \equiv \mathbf{k}_{i},
$$

i.e., set empty sums to zero in the definition (A28). It is easily seen that the Jacobi determinant of this transformation is one. Furthermore the two $\delta$ functions fix

$$
\mathbf{l}_{n}=\left(\frac{\mathbf{q}}{2}, K\right)=\mathbf{K}+\frac{\mathbf{q}}{2} \equiv \mathbf{k}_{f}
$$

We then obtain the final result

$$
\begin{aligned}
T_{n}= & \int \frac{d^{3} l_{n-1}}{(2 \pi)^{3}} \tilde{V}\left(\mathbf{l}_{n}-\mathbf{l}_{n-1}\right) \\
& \times \prod_{k=1}^{n-2}\left(\int \frac{d^{3} l_{k}}{(2 \pi)^{3}} \frac{\tilde{V}\left(\mathbf{l}_{k+1}-\mathbf{l}_{k}\right)}{E-\mathbf{l}_{k}^{2} /(2 m)+i 0}\right) \tilde{V}\left(\mathbf{l}_{1}-\mathbf{l}_{0}\right) \\
= & \int \frac{d^{3} l_{n-1}}{(2 \pi)^{3}} \cdots \frac{d^{3} l_{1}}{(2 \pi)^{3}} \tilde{V}\left(\mathbf{k}_{f}-\mathbf{l}_{n-1}\right) \frac{1}{E-\frac{\mathbf{l}_{n-1}^{2}}{2 m}+i 0}
\end{aligned}
$$

$$
\times \tilde{V}\left(\mathbf{l}_{n-1}-\mathbf{l}_{n-2}\right) \cdots \tilde{V}\left(\mathbf{l}_{2}-\mathbf{l}_{1}\right) \frac{1}{E-\frac{\mathbf{l}_{1}^{2}}{2 m}+i 0} \tilde{V}\left(\mathbf{l}_{1}-\mathbf{k}_{i}\right) .
$$

This is identical with the standard quantum-mechanical expression obtained in time-independent scattering theory (here operators are denoted by a "hat")

$$
\begin{aligned}
T_{n}=\langle & \phi_{f} \mid V(\hat{x}) \frac{1}{E-\hat{p}^{2} /(2 m)+i 0} V(\hat{x}) \cdots V(\hat{x}) \frac{1}{E-\hat{p}^{2} /(2 m)+i 0} \\
& \times V(\hat{x})\left|\phi_{i}\right\rangle
\end{aligned}
$$

when evaluated in momentum space [note the convention (A2) and the normalization of the scattering states $\phi]$.

\section{APPENDIX B: EVALUATION OF FIRST-ORDER CORRECTIONS FOR A SPHERICALLY SYMMETRIC POTENTIAL}

For the eikonal expansion we start from Eq. (73), change to $z=K t / m$ and use $\nabla V(r)=\mathbf{r} V^{\prime}(r) / r$ where the prime indicates differentiation with respect to the argument. This gives

$$
\chi_{\mathrm{AI}}^{(1)}(b)=\frac{m^{2}}{4 K^{3}} \int_{-\infty}^{+\infty} d z_{1} d z_{2} \frac{V^{\prime}\left(r_{1}\right)}{r_{1}} \frac{V^{\prime}\left(r_{2}\right)}{r_{2}}\left[b^{2}+z_{1} z_{2}\right]\left|z_{1}-z_{2}\right| .
$$

Since $r=\sqrt{b^{2}+z^{2}}$ is invariant under $z \rightarrow-z$ and the integrand is symmetric with respect to $z_{1} \leftrightarrow z_{2}$ one obtains

$$
\chi^{(1)}(b)=\frac{2 m^{2}}{K^{3}} \int_{0}^{\infty} d z_{1} z_{1} \frac{V^{\prime}\left(r_{1}\right)}{r_{1}} \int_{0}^{z_{1}} d z_{2} \frac{V^{\prime}\left(r_{2}\right)}{r_{2}}\left[b^{2}-z_{2}^{2}\right] .
$$

The simple relations

$$
z \frac{V^{\prime}(r)}{r}=\frac{\partial V(r)}{\partial z}, \quad b \frac{V^{\prime}(r)}{r}=\frac{\partial V(r)}{\partial b}
$$

can be used to reduce the first-order eikonal phase to onedimensional quadratures. The first one together with an integration by parts leads to

$$
\chi_{\mathrm{AI}}^{(1)}(b)=-\frac{2 m^{2}}{K^{3}} \int_{0}^{\infty} d z V(r) \frac{V^{\prime}(r)}{r}\left[b^{2}-z^{2}\right] .
$$

Then the second relation in Eq. (B3) may be employed to yield

$$
\chi_{\mathrm{AI}}^{(1)}(b)=-\frac{m^{2}}{K^{3}} \int_{0}^{\infty} d z\left[V(r) b \frac{\partial V(r)}{\partial b}-z V(r) \frac{\partial V(r)}{\partial z}\right] .
$$

Finally another integration by parts in the last term gives Eq. (74).

For the first-order ray correction the algebra is a little bit more involved. We start from Eq. (105) and use 


$$
\nabla V(\rho)=\frac{V^{\prime}(\rho)}{\rho}\left[\mathbf{b}+\frac{\mathbf{q}}{2 m}|t|+\frac{\mathbf{K}}{m} t\right] .
$$

Restricting the integration region to positive values one obtains

$$
\begin{aligned}
\chi_{\text {ray }}^{(1)}(\mathbf{b}, \mathbf{q}) & =-\frac{1}{2 m} \int_{0}^{\infty} d t_{1} d t_{2} \frac{V^{\prime}\left(\rho_{1}\right)}{\rho_{1}} \frac{V^{\prime}\left(\rho_{2}\right)}{\rho_{2}} \\
\times & {\left[b^{2}+\frac{\mathbf{b} \cdot \mathbf{q}}{2 m}\left(t_{1}+t_{2}\right)+\frac{k^{2}}{m^{2}} t_{1} t_{2}\right]\left[t_{1}+t_{2}-\left|t_{1}-t_{2}\right|\right] } \\
= & -\frac{2 m^{2}}{k^{3}} \int_{0}^{\infty} d z_{1} \frac{V^{\prime}\left(\rho_{1}\right)}{\rho_{1}} \int_{0}^{z_{1}} d z_{2} z_{2} \frac{V^{\prime}\left(\rho_{2}\right)}{\rho_{2}} \\
& \times\left[b^{2}+\beta b\left(z_{1}+z_{2}\right)+z_{1} z_{2}\right],
\end{aligned}
$$

where in the last line the transformation $z=k t / m$ and the symmetry of the integrand have been used. $\beta$ is defined in Eq. (86). As in the eikonal case we would like to reduce this expression to one-dimensional integrals but the derivatives of the potential with respect to $z, b$ are now more complicated:

$$
\frac{\partial V(\rho)}{\partial z}=\frac{V^{\prime}(\rho)}{\rho}(\beta b+z), \quad \frac{\partial V(\rho)}{\partial b}=\frac{V^{\prime}(\rho)}{\rho}(b+\beta z) .
$$

Solving for $z V^{\prime} / \rho$ and $b V^{\prime} / \rho$ one now has instead of the relations (B3)

$$
\begin{aligned}
& z \frac{V^{\prime}(\rho)}{\rho}=\frac{1}{1-\beta^{2}}\left[\frac{\partial V}{\partial z}-\beta \frac{\partial V}{\partial b}\right], \\
& b \frac{V^{\prime}(\rho)}{\rho}=\frac{1}{1-\beta^{2}}\left[\frac{\partial V}{\partial b}-\beta \frac{\partial V}{\partial z}\right] .
\end{aligned}
$$

As a final combination one needs

$$
z^{2} \frac{V^{\prime}(\rho)}{\rho}=z \frac{\partial V}{\partial z}-\frac{\beta b}{1-\beta^{2}}\left[\frac{\partial V}{\partial z}-\beta \frac{\partial V}{\partial b}\right] .
$$

Equation (B7) multiplied by $-k^{3} /\left(2 m^{2}\right)$ consists of four terms which can be simplified with the help of the above relations and appropriate integrations by part. The first term is

$$
\begin{aligned}
& b^{2} \int_{0}^{\infty} d z_{1} \frac{V^{\prime}\left(\rho_{1}\right)}{\rho_{1}} \int_{0}^{z_{1}} d z_{2} z_{2} \frac{V^{\prime}\left(\rho_{2}\right)}{\rho_{2}}=\frac{b}{\left(1-\beta^{2}\right)^{2}} \int_{0}^{\infty} d z_{1}\left(\frac{\partial V_{1}}{\partial b}-\beta \frac{\partial V}{\partial z_{1}}\right) \int_{0}^{z_{1}} d z_{2}\left(\frac{\partial V}{\partial z_{2}}-\beta \frac{\partial V_{2}}{\partial b}\right) \\
& =\frac{b}{\left(1-\beta^{2}\right)^{2}} \int_{0}^{\infty} d z_{1}\left[\frac{\partial V_{1}}{\partial b}\left[V_{1}-V(b)\right]-\frac{\beta}{2} \frac{d}{d z_{1}}\left(\int_{0}^{z_{1}} d z_{2} \frac{\partial V_{2}}{\partial b}\right)^{2}-\beta \frac{\partial V_{1}}{\partial z_{1}}\left[V_{1}-V(b)\right]+\beta^{2} \frac{\partial V}{\partial z_{1}} \int_{0}^{z_{1}} d z_{2} \frac{\partial V_{2}}{\partial b}\right] \\
& =\frac{1}{2} \frac{b}{\left(1-\beta^{2}\right)^{2}}\left[\left(1-\beta^{2}\right) \frac{\partial}{\partial b} \int_{0}^{\infty} d z V^{2}-\beta V^{2}(b)-2 V(b) \frac{\partial}{\partial b} \int_{0}^{\infty} d z V-\beta\left(\frac{\partial}{\partial b} \int_{0}^{\infty} d z V\right)^{2}\right] .
\end{aligned}
$$

The second one reads

$$
\beta b \int_{0}^{\infty} d z_{1} z_{1} \frac{V^{\prime}\left(\rho_{1}\right)}{\rho_{1}} \int_{0}^{z_{1}} d z_{2} z_{2} \frac{V^{\prime}\left(\rho_{2}\right)}{\rho_{2}}=\beta b \int_{0}^{\infty} d z_{1} \frac{1}{2} \frac{d}{d z_{1}}\left(\int_{0}^{z_{1}} d z_{2} z_{2} \frac{V^{\prime}\left(\rho_{2}\right)}{\rho_{2}}\right)^{2}=\frac{1}{2} \frac{\beta b}{\left(1-\beta^{2}\right)^{2}}\left(-V(b)-\beta \frac{\partial}{\partial b} \int_{0}^{\infty} d z V\right)^{2} .
$$

Finally the third and fourth terms combined give

$$
\begin{aligned}
& \beta b \int_{0}^{\infty} d z_{1} \frac{V^{\prime}\left(\rho_{1}\right)}{\rho_{1}} \int_{0}^{z_{1}} d z_{2} z_{2}^{2} \frac{V^{\prime}\left(\rho_{2}\right)}{\rho_{2}}+\int_{0}^{\infty} d z_{1} z_{1} \frac{V^{\prime}\left(\rho_{1}\right)}{\rho_{1}} \int_{0}^{z_{1}} d z_{2} z_{2}^{2} \frac{V^{\prime}\left(\rho_{2}\right)}{\rho_{2}}=\int_{0}^{\infty} d z_{1} \frac{\partial V}{\partial z_{1}} \int_{0}^{z_{1}} d z_{2} z_{2}^{2} \frac{V^{\prime}\left(\rho_{2}\right)}{\rho_{2}}=-\int_{0}^{\infty} d z V z^{2} \frac{V^{\prime}(\rho)}{\rho} \\
& \quad=\frac{1}{2} \int_{0}^{\infty} d z V^{2}-\frac{1}{2} \frac{\beta b}{1-\beta^{2}} V^{2}(b)-\frac{1}{2} \frac{\beta^{2} b}{1-\beta^{2}} \frac{\partial}{\partial b} \int_{0}^{\infty} d z V^{2} .
\end{aligned}
$$

Summing up all contributions we obtain the result given in Eq. (106).

\section{APPENDIX C: EIKONAL AND RAY PHASES FOR A GAUSSIAN POTENTIAL}

Here we list the analytical expressions for the phases of a Gaussian potential

$$
V(r)=V_{0} e^{-\alpha^{2} r^{2}}, \quad \alpha=\frac{1}{R} .
$$

For the AI eikonal expansion we have the well-known results [14]

$$
\chi_{\mathrm{AI}}^{(0)}(b)=-\frac{m V_{0}}{K} \frac{\sqrt{\pi}}{\alpha} e^{-\alpha^{2} b^{2}}
$$




$$
\chi_{\mathrm{AI}}^{(1)}(b)=-\frac{1}{2 K}\left(\frac{m V_{0}}{K}\right)^{2} \sqrt{\frac{\pi}{2}} \frac{1}{\alpha}\left(1-4 \alpha^{2} b^{2}\right) e^{-2 \alpha^{2} b^{2}} .
$$

For the ray expansion we get in zeroth order

$$
\chi_{\text {ray }}^{(0)}(b, \beta)=-\frac{m V_{0}}{k} \frac{\sqrt{\pi}}{\alpha} e^{-\alpha^{2} b^{2}} F(B),
$$

where

$$
F(B=\alpha b \beta):=e^{B^{2}} \operatorname{erfc}(B), \quad F(0)=1
$$

and $\operatorname{erfc}(x)=1-\operatorname{erf}(x)$ is the complimentary error function [47]. The correction terms are:

$$
\begin{aligned}
\chi_{\text {ray }}^{(1)}(b, \beta) & =-\frac{1}{2 k}\left(\frac{m V_{0}}{k}\right)^{2} \sqrt{\frac{\pi}{2}} \frac{1}{\alpha} e^{-2 \alpha^{2} b^{2}} \\
\times & \left\{\left[1-4 \alpha^{2} b^{2}\left(1-\beta^{2}\right)\right] F(\sqrt{2} B)\right. \\
+ & \left.2 \sqrt{2} \alpha^{2} b^{2}\left(1-\beta^{2}\right) F(B)[2-\sqrt{\pi} B F(B)]\right\}, \\
\omega_{\text {ray }}^{(1)}(b, \beta, \theta) & =\frac{2}{k} \frac{m V_{0}}{k} e^{-\alpha b^{2}}\left\{\cos ^{2} \frac{\theta}{2}-\alpha^{2} b^{2}\right. \\
+ & B^{2}\left(2-\sin ^{2} \frac{\theta}{2}\right)-\sqrt{\pi} B F(B)\left[2-\frac{3}{2} \sin ^{2} \frac{\theta}{2}-\alpha^{2} b^{2}\right. \\
+ & \left.\left.B^{2}\left(2-\sin ^{2} \frac{\theta}{2}\right)\right]\right\} .
\end{aligned}
$$

We have checked these formulae by performing the integrals with MAPLE and by direct numerical integration of Eqs. (B7) and (95) for a Gaussian potential.
[1] L. S. Rodberg and R. M. Thaler, Introduction to the Quantum Theory of Scattering (Academic Press, New York, 1967); R. G. Newton, Scattering Theory of Waves and Particles, 2nd ed. (Springer, New York, 1982).

[2] L. S. Schulman, Techniques and Applications of Path Integration (Wiley, New York, 1981).

[3] E. Gildener and A. Patrascioiu, Phys. Rev. D 16, 423 (1977).

[4] E. B. Manoukian, Quantum Theory: A Wide Spectrum (Springer, Dordrecht, 2006), Chap 15.6.

[5] E. B. Manoukian and S. Sukkhasena, Prog. Theor. Phys. 116, 795 (2006).

[6] C. Itzykson and J.-B. Zuber, Quantum Field Theory (McGraw Hill, New York, 1980), Chap 9.1.1.

[7] J. Hughes, Ann. Phys. 205, 418 (1991).

[8] N. Makri, J. Math. Phys. 36, 2430 (1995).

[9] B. Burghardt and J. Stolze, J. Phys. A 32, 2075 (1999).

[10] S. Lenz and H. Mall, Comput. Phys. Commun. 90, 215 (1995); J. Koch, H. Mall, and S. Lenz, Comput. Phys. Commun. 108, 115 (1998).

[11] N. Makri, Comput. Phys. Commun. 63, 389 (1991); B. L. G. Bakker, M. I. Polikarpov, and L. V. Shevchenko (unpublished). Recently, the discretized Feynman path integral was numerically evaluated with a wave-packet description and Gaussian expansion methods in Ref. [12]. Stochastic quantization techniques with an additional "Langevin time" have been used for real-time simulations of interacting (gauge) fields by Berges and collaborators: J. Berges and D. Sexty, Nucl. Phys. B 799, 306 (2008); J. Berges, S. Borsanyi, D. Sexty, and I. O. Stamatescu, Phys. Rev. D 75, 045007 (2007).

[12] V. V. Smirnov, Phys. Rev. A 76, 052706 (2007).

[13] C. Elster, T. Lin, W. Glöckle, and S. Jeschonnek, arXiv:0805.2010 [nucl-th].

[14] S. J. Wallace, Ann. Phys. 78, 190 (1973).

[15] S. Sarkar, Phys. Rev. D 21, 3437 (1980).

[16] N. V. Bondarenko and N. F. Shulga, Phys. Lett. B 427, 114 (1998); T. W. Chen, Phys. Rev. D 29, 1839 (1984); Phys. Rev. C 30, 585 (1984); M. Kh. Khankhasaev, Theor. Math. Phys.
85, 1139 (1991); A. L. Zubarev and M. M. Musakhanov, Sov. J. Nucl. Phys. 23, 42 (1976).

[17] M. Levy and J. Sucher, Phys. Rev. 186, 1656 (1969).

[18] W. B. Campbell, P. Finkler, C. E. Jones, and M. N. Misheloff, Phys. Rev. D 12, 2363 (1975).

[19] A. V. Bogdanov and G. V. Dubrovskii, Theor. Math. Phys. 30, 146 (1977).

[20] A. V. Vasil'ev and A. V. Kuz'menko, Theor. Math. Phys. 31, 479 (1977).

[21] R. Rosenfelder, Pfadintegrale in der Quantenphysik (in German), https://e-collection.ethbib.ethz.ch/view/eth:26733 [enlarged and corrected version (2003)].

[22] H. Kleinert, Path Integrals in Quantum Mechanics, Statistics, Polymer Physics and Financial Markets, 3rd ed. (World Scientific, Singapore, 2004), Chaps. 2.23 and 2.24.

[23] D. M. Gitman and Sh. M. Shvartsman, e-print arXiv:9310074; Phys. Lett. B 318, 122 (1993); 331, 449(E) (1994); W. da Cruz, J. Phys. A 30, 5225 (1997).

[24] In the Hilbert-space formulation [see R. Rosenfelder and A. W. Schreiber, Eur. Phys. J. C 25, 139 (2002)] we simply have $\left.\mid \mathbf{x})=\frac{1}{2} S \mid \mathbf{v}\right)$.

[25] Assuming that the potential falls off rapidly enough at infinity so that boundary terms do not contribute.

[26] B. Grinstein and D. O'Connell, Phys. Rev. D 78, 105005 (2008); B. Grinstein, D. O'Connell, and M. B. Wise, ibid. 77, 025012 (2008); 77, 065010 (2008); T. D. Lee and G. C. Wick, Nucl. Phys. B 9, 209 (1969); Phys. Rev. D 2, 1033 (1970).

[27] A. van Tonder and M. Dorca, Int. J. Mod. Phys. A 22, 2563 (2007).

[28] The large values of $\tau$ needed to generate the $\delta$ function obviously violate the requirement $|\tau| \ll T$ used for showing the invariance of the action. Consequently there can be no strict energy conservation for large but finite $T$.

[29] This is the typical situation for high-energy scattering in a potential of finite range since otherwise the term $\mathrm{Kt} / \mathrm{m}$ in the argument of the potential would exceed the range. In principle, this could be cancelled by the term $\mathbf{x}_{v}(t)$, but this would re- 
quire very large velocity fluctuations which are suppressed by the free action.

[30] Equation (11) is a special case of this relation.

[31] T. Adachi and T. Kotani, Prog. Theor. Phys. 39, 430 (1968); 39, 785 (1968); M. M. Islam, in Boulder 1967, Lectures In Theoretical Physics Vol. Xb, edited by A. O. Barut and W. E. Brittin (Interscience, New York, 1968), p. 97.

[32] A. Messiah, Quantum Mechanics (North Holland, Amsterdam, 1965), Vol. 2, pp. 807, 831, 867.

[33] Here it is essential that $\mathbf{K}$ is multiplied by $t$ but $\mathbf{q}$ by $|t|$. Some works $[4,5,22]$ have chosen the initial or final momentum as preferred direction which does not exhibit the microreversibility of the $T$ matrix in a simple way.

[34] The superscript on the phase denotes the corresponding order in powers of $K^{-1}$ while $m / K$ is fixed.

[35] H. D. I. Abarbanel and C. Itzykson, Phys. Rev. Lett. 23, 53 (1969).

[36] L. E. Reichl, A Modern Course in Statistical Physics (University of Texas Press, Austin, 1987), pp. 143, 144; M. Le Bellac, Quantum and Statistical Field Theory (Oxford Science Publications, Oxford 1991), pp. 171, 172; R. Rosenfelder, Ann. Phys. 128, 188 (1980).
[37] Again superscripts denote the expansion order, this time in $k^{-1}$.

[38] T. Matsuki, Prog. Theor. Phys. 55, 751 (1976).

[39] T. J. De Forest and J. D. Walecka, Am. J. Optom. Physiol. Opt. 15, 1 (1966); P. Guèye et al., Phys. Rev. C 60, 044308 (1999).

[40] A. Baker, Phys. Rev. 134, B240 (1964); D. R. Yennie, F. L. Boos, and D. G. Ravenhall, ibid. 137, B882 (1965); R. Rosenfelder, Z. Phys. A 274, 267 (1975).

[41] Note the logarithmic scale for the cross section in contrast to the linear scale used in Ref. [12].

[42] H. Kröger, Phys. Rep. 210, 45 (1992); J. Holz and W. Glöckle, Phys. Rev. C 37, 1386 (1988); R. Girard, H. Kröger, P. Labelle, and Z. Bajzer, Phys. Rev. A 37, 3195 (1988).

[43] S. J. Wallace, Phys. Rev. D 9, 406 (1974).

[44] J. M. Eisenberg and D. S. Koltun, Theory of Meson Interactions with Nuclei (J. Wiley, New York, 1980), chap. 4.

[45] N. S. Han and N. N. Xuan, e-print arXiv:0804.3432 [quantph]; S. J. Wallace and J. A. McNeil, Phys. Rev. D 16, 3565 (1977).

[46] R. Rosenfelder (unpublished).

[47] M. Abramowitz and I. Stegun (eds.), Handbook of Mathematical Functions (Dover, New York, 1965). 